\title{
Moderate Deviations for the SSEP with a Slow Bond
}

\author{
Xiaofeng Xue *and Linjie Zhao ${ }^{\dagger}$
}

\begin{abstract}
We consider the one dimensional symmetric simple exclusion process with a slow bond. In this model, particles cross each bond at rate $N^{2}$, except one particular bond, the slow bond, where the rate is $N$. Above, $N$ is the scaling parameter. This model has been considered in the context of hydrodynamic limits, fluctuations and large deviations. We investigate moderate deviations from hydrodynamics and obtain a moderate deviation principle.
\end{abstract}

Keywords: exclusion process, slow bond, moderate deviation, exponential martingale.

\section{Introduction}

The symmetric simple exclusion process (SSEP) with a slow bond was introduced in [5] by Franco, Gonçalves and Neumann to derive from microscopic systems PDEs with boundary conditions, which has become a popular topic recently $[1,8,11]$. The process evolves on the discrete ring with $N$ sites, where $N$ is the scaling parameter. There is at most one particle per site. Particles cross each bond at rate $N^{2}$ except one particular bond, where the rate is $N$.

The hydrodynamic limit of the SSEP with a slow bond has been well understood [5,7]. The hydrodynamic equation turns out to be the heat equation with Robin's boundary conditions:

$$
\begin{cases}\partial_{t} \rho(t, u)=\partial_{u}^{2} \rho(t, u), & t>0, u \in \mathbb{T} \backslash\{0\} \\ \partial_{u} \rho\left(t, 0^{+}\right)=\partial_{u} \rho\left(t, 0^{-}\right)=\rho\left(t, 0^{+}\right)-\rho\left(t, 0^{-}\right), & t>0, \\ \rho(0, u)=\gamma(u), & u \in \mathbb{T}\end{cases}
$$

where $\mathbb{T}$ is the continuous ring, $0^{+}$and $0^{-}$denote respectively the right limit and left limit at site 0 , and $\gamma(\cdot)$ is the initial density profile. Then it is natural to consider the equilibrium fluctuations and large deviations from the hydrodynamic limit. Equilibrium fluctuations have been studied in [6] and large deviations in [10] by Franco, Gonçalves and Neumann.

To better understand the SSEP with a slow bond, we consider the moderate deviations from the hydrodynamic limit, which gives asymptotic behavior of the model between the central limit theorem and the large deviation. As far as we know, the only paper concerned about moderate deviations from hydrodynamics is [13] authored by Gao and Quastel, where the classic SSEP was considered. For literatures about theories of moderate deviations, see References [2-4,12,17-19] and so on.

Next, we introduce the SSEP with a slow bond and main results. The process evolves on $\mathbb{T}_{N}=\{0,1, \ldots, N-1\}$ the ring with $N$ sites, with the convention $N \equiv 0$. Therefore, the state

\footnotetext{
*E-mail: xfxue@bjtu.edu.cn Address: School of Science, Beijing Jiaotong University, Beijing 100044, China.

${ }^{\dagger}$ E-mail: zhaolinjie@pku.edu.cn Address: School of Mathematical Sciences, Peking University, Beijing 100871, China.
} 
space is $\{0,1\}^{\mathbb{T}_{N}}$. For each configuration $\eta \in\{0,1\}^{\mathbb{T}_{N}}, \eta(x)=1$ means site $x$ is occupied by a particle, and $\eta(x)=0$ means site $x$ is vacant. The infinitesimal generator $\mathcal{L}_{N}$ of the process is

$$
\mathcal{L}_{N} f(\eta)=N\left[f\left(\eta^{-1,0}\right)-f(\eta)\right]+N^{2} \sum_{\substack{x \in \mathbb{T}_{N} \\ x \neq-1}}\left[f\left(\eta^{x, x+1}\right)-f(\eta)\right],
$$

where

$$
\eta^{x, y}(u)= \begin{cases}\eta(u) & \text { if } u \neq x, y \\ \eta(y) & \text { if } u=x \\ \eta(x) & \text { if } u=y\end{cases}
$$

for any $x \neq y$. Denote by $\left\{\eta_{t}\right\}_{t \geq 0}$ the process with generator $\mathcal{L}_{N}$. We suppress the dependence of the process $\left\{\eta_{t}\right\}_{t>0}$ on $N$ for short.

Equivalently, we can define the process in the following way. For each $i \neq-1$, let $\left\{Y_{i}(t)\right\}_{t \geq 0}$ be a Poisson process with rate $N^{2}$ and $\left\{Y_{-1}(t)\right\}_{t \geq 0}$ be a Poisson process with rate $N$. Assume that all these Poisson processes are independent. Then at any event moment of $Y_{i}(\cdot), \eta(i)$ and $\eta(i+1)$ exchange their values.

The SSEP with a slow bond has a family of invariant measures indexed by the particle density. To be precise, let $\nu_{\rho}, \rho \in[0,1]$, be the product measure on $\mathbb{T}_{N}$ with marginals given by

$$
\nu_{\rho}\{\eta: \eta(x)=1\}=\rho, \forall x \in \mathbb{T}_{N} .
$$

Then, it can be checked easily that $\nu_{\rho}, \rho \in[0,1]$, are reversible measures for the process $\left\{\eta_{t}\right\}_{t \geq 0}$.

To define the empirical density and rate functions, we need to introduce some definitions and notations and then discuss some topological issues. We identify $\mathbb{T}$ with $[0,1)$, and thus $0^{+}$with 0 and $0^{-}$with 1 . By the boundary conditions imposed on the hydrodynamic equation (1.1), it is reasonable to consider test functions $G \in C^{1}[0,1]$ with the property

$$
G^{\prime}(0)=G^{\prime}(1)=G(0)-G(1) .
$$

The result of this paper relies heavily on the above kind of functions, especially trigonometric functions satisfying (1.2). Define $\mathscr{G}_{0}$ as

$$
\mathscr{G}_{0}:=\operatorname{span}\left(\left\{\sin \left(k_{n}\left(x-\frac{1}{2}\right)\right)\right\}_{n \geq 1} \bigcup\{\cos (2 n \pi x)\}_{n \geq 0}\right),
$$

where $k_{n}$ is the unique solution to the equation $-\frac{x}{2}=\tan \frac{x}{2}$ in $((2 n-1) \pi,(2 n+1) \pi)$ for each $n \geq 1$. It can be checked easily that any $G \in \mathscr{G}_{0}$ satisfies (1.2). According to [9, Theorem 1] given by Franco and Landim, we can prove the set of the above trigonometric functions is a basis in $L^{2}[0,1]$, which is crucial to construct the topology of this paper.

Lemma 1.1. The set $\left\{\sin \left(k_{n}(x-1 / 2)\right)\right\}_{n \geq 1} \bigcup\{\cos (2 n \pi x)\}_{n \geq 0}$ is an orthogonal basis of $L^{2}[0,1]$.

We put the proof of Lemma 1.1 in the appendix.

Let $\mathscr{M}$ be the space of linear (not necessarily bounded) functionals on $\mathscr{G}_{0}$ endowed with the following topology: for any $\mathscr{A}_{n} \in \mathscr{M}, n \geq 1$, and $\mathscr{A} \in \mathscr{M}$,

$$
\lim _{n \rightarrow+\infty} \mathscr{A}_{n}=\mathscr{A} \quad \text { in } \mathscr{M} \quad \text { if and only if } \quad \lim _{n \rightarrow+\infty} \mathscr{A}_{n}\left(\theta_{k}\right)=\mathscr{A}\left(\theta_{k}\right) \quad \text { for all integers } k,
$$


where $\theta_{n}(x)=\sin \left(k_{n}(x-1 / 2)\right)$ for $n \geq 1$ and $\theta_{-n}(x)=\cos (2 n \pi x)$ for $n \geq 0$. The above topology is metrizable and the metric $d(\cdot, \cdot)$ is given by

$$
d\left(\mathscr{A}_{1}, \mathscr{A}_{2}\right)=\sum_{-\infty<n<+\infty} \frac{1}{2^{|n|}} \frac{\left|\mathscr{A}_{1}\left(\theta_{n}\right)-\mathscr{A}_{2}\left(\theta_{n}\right)\right|}{1+\left|\mathscr{A}_{1}\left(\theta_{n}\right)-\mathscr{A}_{2}\left(\theta_{n}\right)\right|}, \quad \mathscr{A}_{1}, \mathscr{A}_{2} \in \mathscr{M} .
$$

It can be checked directly that the space $\mathscr{M}$ is complete and separable under the above metric. Note that a bounded signed measure $\mu$ on $[0,1]$ can be identified with an element in $\mathscr{M}$ in the sense that $\mu(f)=\int_{[0,1]} f(x) \mu(d x)$ for any $f \in \mathscr{G}_{0}$.

Remark 1.2. We construct the above topology for technical reasons. Mainly, we can not show the uniqueness or existence of the weak solution to a PDE arising from hydrodynamics of the SSEP with a slow bond under a Girsanov's transformed measure. However, if we do not distinguish two measures $\mu_{1}$ and $\mu_{2}$ satisfying $\mu_{1}\left(\theta_{n}\right)=\mu_{2}\left(\theta_{n}\right)$ for all $n$, the above PDE can be reduced to an ODE on $\mathscr{M}$, the existence and uniqueness of the solution to which can be rigorously proved. For mathematical details, see Section 4 and appendix.

In the following, we will fix a horizonal time $T>0$. Let $D([0, T], \mathscr{M})$ be the space of càdlàg functions from $[0, T]$ to $\mathscr{M}$ endowed with the Skorohod topology. Define the rescaled central empirical density $\mu_{t}^{N}(d u)$ as

$$
\mu_{t}^{N}(d u):=\frac{1}{a_{N}} \sum_{x \in \mathbb{T}_{N}}\left(\eta_{t}(x)-\rho\right) \delta_{x / N}(d u),
$$

where $\sqrt{N} \ll a_{N} \ll N$. We will regard $\mu^{N}:=\left\{\mu_{t}^{N}\right\}_{0 \leq t \leq T}$ as a random element taking values in $D([0, T], \mathscr{M})$.

Let $\mathscr{G}$ be the family of functions $G:[0, T] \times[0,1] \rightarrow \mathbb{R}$ with the following forms: there exist $M \in \mathbb{N}$ and $b_{m}(t) \in C^{1}([0, T]),-M \leq m \leq M$ such that

$$
G(t, u)=\sum_{m=-M}^{M} b_{m}(t) \theta_{m}(u), \quad(t, u) \in[0, T] \times[0,1] .
$$

Then for any $G \in \mathscr{G}$,

$$
\partial_{u} G(t, 0)=\partial_{u} G(t, 1)=G(t, 0)-G(t, 1), \quad \forall t \in[0, T] .
$$

We sometimes write $G_{t}(u)$ for $G(t, u)$. For $G \in \mathscr{G}$, define the extended Laplacian $\tilde{\Delta}$ as

$$
\tilde{\Delta} G_{t}(u)= \begin{cases}\partial_{u}^{2} G_{t}(u) & \text { if } u \neq 0 \\ \partial_{u}^{2} G_{t}\left(0^{+}\right) & \text {if } u=0\end{cases}
$$

Fix a density $\rho \in(0,1)$. Denote by $Q_{\rho}^{N}$ the law of $\left\{\mu_{t}^{N}\right\}_{0 \leq t \leq T}$ with initial distribution $\nu_{\rho}$. Let $\mathbb{P}_{\rho}^{N}$ be the law of the process $\left\{\eta_{t}\right\}_{0 \leq t \leq T}$ with initial distribution $\nu_{\rho}$, and $\mathbb{E}_{\rho}^{N}$ the corresponding 
expectation. Let $\mathrm{E}_{\nu_{\rho}}$ be the expectation with respect to $\nu_{\rho}$. For $\mu \in D([0, T], \mathscr{M})$, define

$$
\begin{aligned}
& I(\mu):=I_{\text {ini }}\left(\mu_{0}\right)+I_{\text {dyn }}(\mu), \\
& I_{\text {ini }}\left(\mu_{0}\right):=\sup _{\gamma \in \mathscr{G}_{0}}\left\{\mu_{0}(\gamma)-\frac{\rho(1-\rho)}{2} \int_{0}^{1} \gamma^{2}(u) d u\right\}, \\
& I_{d y n}(\mu):=\sup _{G \in \mathscr{G}}\left\{\ell_{T}(\mu, G)-\rho(1-\rho) \int_{0}^{T}\left(G_{t}(0)-G_{t}(1)\right)^{2} d t\right. \\
&\left.\quad-\rho(1-\rho) \int_{0}^{T} \int_{0}^{1}\left(\partial_{u} G_{t}(u)\right)^{2} d u d t\right\},
\end{aligned}
$$

where

$$
\ell_{T}(\mu, G):=\mu_{T}\left(G_{T}\right)-\mu_{0}\left(G_{0}\right)-\int_{0}^{T} \mu_{t}\left(\left(\partial_{t}+\tilde{\Delta}\right) G_{t}\right) d t
$$

Now we are ready to state the main result of the paper.

Theorem 1.3. For any closed set $C$ of $D([0, T], \mathscr{M})$,

$$
\limsup _{N \rightarrow \infty} \frac{N}{a_{N}^{2}} \log Q_{\rho}^{N}[C] \leq-\inf _{\mu \in C} I(\mu),
$$

and for any open set $O$ of $D([0, T], \mathscr{M})$,

$$
\liminf _{N \rightarrow \infty} \frac{N}{a_{N}^{2}} \log Q_{\rho}^{N}[O] \geq-\inf _{\mu \in O} I(\mu) .
$$

Remark 1.4. We recall the large deviation principle of the SSEP with a slow bond established in [10] by Franco and Neumann for a comparison. Note that definitions and notations in this remark are not utilized elsewhere. Let

$$
\pi_{t}^{N}(d u)=\frac{1}{N} \sum_{x \in \mathbb{T}_{N}} \eta_{t}(x) \delta_{x / N}(d u), \quad \pi^{N}=\left\{\pi_{t}^{N}\right\}_{0 \leq t \leq T},
$$

then it was shown in [10] that, roughly speaking,

$$
P\left(\pi^{N} \approx \pi\right) \approx \exp \{-N J(\pi)\}
$$

assuming uniqueness for the weak solution to hydrodynamic equation associated to the perturbed process, where

$$
J(\pi)=\sup _{H \in C^{1,2}([0, T] \times[0,1])}\left\{\widehat{\ell}_{H}(\pi)-\Phi_{H}(\pi)\right\}
$$

with $\widehat{\ell}_{H}(\pi)$ given by

$$
\begin{aligned}
\widehat{\ell}_{H}(\pi)= & \left\langle\rho_{T}, H_{T}\right\rangle-\left\langle\rho_{0}, H_{0}\right\rangle-\int_{0}^{T}\left\langle\rho_{t},\left(\partial_{t}+\Delta\right) H_{t}\right\rangle d t \\
& -\int_{0}^{T}\left\{\rho_{t}(0) \partial_{u} H_{t}(0)-\rho_{t}(1) \partial_{u} H_{t}(1)\right\} d t+\int_{0}^{T}\left(\rho_{t}(0)-\rho_{t}(1)\right) \delta H_{t}(0) d t
\end{aligned}
$$


and $\Phi_{H}(\pi)$ given by

$$
\begin{aligned}
\Phi_{H}(\pi)= & \int_{0}^{T}\left\langle\chi\left(\rho_{t}\right),\left(\partial_{u} H_{t}\right)^{2}\right\rangle d t+\int_{0}^{T} \rho_{t}(1)\left(1-\rho_{t}(0)\right) \psi\left(\delta H_{t}(0)\right) d t \\
& +\int_{0}^{T} \rho_{t}(0)\left(1-\rho_{t}(1)\right) \psi\left(-\delta H_{t}(0)\right) d t,
\end{aligned}
$$

where $\rho_{t}$ is the Radon-Nikodym derivative of $\pi_{t}$ with respect to the Lebesgue measure, $\psi(x)=$ $e^{x}-x-1, \chi(\rho)=\rho(1-\rho)$ and $\delta H_{t}(0)=H_{t}(0)-H_{t}(1)$. Since $e^{x}-x-1=\frac{x^{2}}{2}+o\left(x^{2}\right)$ as $|x|$ decreases to 0 and $\widehat{\ell}_{H}(\pi)$ equals $\ell_{T}(\pi, H)$ when $H$ satisfies (1.3), the rate function $I_{d y n}$ can be intuitively considered as the quadratic part of $J$ about its minimum, which is a common relationship between large and moderate deviations for many models in statistical physics.

Notation. For deterministic positive sequences $\left\{b_{n}\right\}_{n \geq 1},\left\{c_{n}\right\}_{n \geq 1}$ and random sequence $\left\{X_{n}\right\}_{n \geq 1}$, we write $b_{n}=o\left(c_{n}\right)$ if $\limsup _{n \rightarrow \infty} b_{n} / c_{n}=0$ and $b_{n}=\mathcal{O}\left(c_{n}\right)$ if $\limsup _{n \rightarrow \infty} b_{n} / c_{n}<C$ for some constant $C$ independent of $n$. We also write $b_{n}=\mathcal{O}_{G}\left(c_{n}\right)$ to stress the dependence on some parameter $G$ of the constant $C$. We write $X_{n}=o_{p}\left(c_{n}\right)$ if $X_{n} / c_{n} \rightarrow 0$ in probability as $n \rightarrow \infty$, and $X_{n}=o_{\exp }\left(c_{n}\right)$ if

$$
\limsup _{n \rightarrow+\infty} \frac{1}{c_{n}} \log P\left(\left|X_{n}\right|>\epsilon\right)=-\infty, \quad \forall \epsilon>0 .
$$

We remark on these last points that the constant throughout the paper may be different from line to line.

The rest of the paper is devoted to the proof of Theorem 1.3. In Section 2 we give several super-exponential estimates that are necessary in the proof of upper and lower bounds as a preparation. Moderate upper bounds are proved in Section 3. Our proof follows a strategy similar with that introduced in [13], except for some details modified due to technical reasons caused by the slow bond. First, as introduced above, we have to choose a proper topology and to consider the empirical density as a random element taking values in the linear functional space $\mathscr{M}$, instead of the dual of Schwartz functions. Second, an extra super-exponential estimate (Lemma 2.1) is needed. Third, because of the topology constructed, we have to use a different version of Minimax Theorem (Theorem 3.2) from the one in [13]. Moderate lower bounds are proved in Section 4. A crucial step in the proof is the utilizing of a generalized Girsanov's theorem to give the hydrodynamic equation of the model under a transformed measure.

\section{Super-exponential Decay}

In this section, we mainly present three super-exponential estimates that are critical when making some replacements and proving exponential tightness.

Lemma 2.1. For any continuous function $G:[0, T] \rightarrow \mathbb{R}$ and any $\delta, t>0$,

$$
\limsup _{N \rightarrow \infty} \frac{1}{a_{N}} \log \mathbb{P}_{\rho}^{N}\left[\left|\int_{0}^{t}\left(\eta_{s}(0)\left(1-\eta_{s}(-1)\right)-\rho(1-\rho)\right) G_{s} d s\right|>\delta\right]=-\infty .
$$

The same result holds with $\eta_{s}(0)\left(1-\eta_{s}(-1)\right)$ replaced by $\eta_{s}(-1)\left(1-\eta_{s}(0)\right)$. 
Proof. We only present the proof of (2.1) since the rest is the same. For any integer $M>0$ and $x \in \mathbb{T}_{N}$, define $\eta^{M, \mathrm{R}}(x)$ (resp. $\eta^{M, \mathrm{~L}}(x)$ ) as the average density over the box of size $M$ to the right (resp. left) of site $x$,

$$
\eta^{M, \mathrm{R}}(x)=\frac{1}{M} \sum_{y=x}^{x+M-1} \eta(y), \quad \eta^{M, \mathrm{~L}}(x)=\frac{1}{M} \sum_{y=x-M+1}^{x} \eta(y) .
$$

Note that for every integer $M>0$,

$$
\begin{aligned}
\eta(0)(1-\eta(-1)) & -\rho(1-\rho)=\left(\eta(0)-\eta^{M, \mathrm{R}}(0)\right)(1-\eta(-1)) \\
& +\eta^{M, \mathrm{R}}(0)\left(\eta^{M, \mathrm{~L}}(-1)-\eta(-1)\right)+\left(\eta^{M, \mathrm{R}}(0)-\rho\right)\left(1-\eta^{M, \mathrm{~L}}(-1)\right) \\
& +\rho\left(\rho-\eta^{M, \mathrm{~L}}(-1)\right) .
\end{aligned}
$$

Since for any positive sequences $\left\{b_{N}\right\}_{N \geq 1}$ and $\left\{c_{N}\right\}_{N \geq 1}$,

$$
\limsup _{N \rightarrow \infty} \frac{1}{a_{N}} \log \left(b_{N}+c_{N}\right) \leq \max \left\{\limsup _{N \rightarrow \infty} \frac{1}{a_{N}} \log b_{N}, \limsup _{N \rightarrow \infty} \frac{1}{a_{N}} \log c_{N}\right\},
$$

to prove (2.1), we only need to prove for any $\delta>0$,

$$
\begin{gathered}
\limsup _{N \rightarrow \infty} \frac{1}{a_{N}} \log \mathbb{P}_{\rho}^{N}\left[\left|\int_{0}^{t}\left(\eta_{s}(0)-\eta_{s}^{M, \mathrm{R}}(0)\right)\left(1-\eta_{s}(-1)\right) G_{s} d s\right|>\delta\right]=-\infty, \\
\limsup _{N \rightarrow \infty} \frac{1}{a_{N}} \log \mathbb{P}_{\rho}^{N}\left[\left|\int_{0}^{t} \eta_{s}^{M, \mathrm{R}}(0)\left(\eta_{s}^{M, \mathrm{~L}}(-1)-\eta_{s}(-1)\right) G_{s} d s\right|>\delta\right]=-\infty, \\
\limsup _{N \rightarrow \infty} \frac{1}{a_{N}} \log \mathbb{P}_{\rho}^{N}\left[\left|\int_{0}^{t}\left(\eta_{s}^{M, \mathrm{R}}(0)-\rho\right)\left(1-\eta_{s}^{M, \mathrm{~L}}(-1)\right) G_{s} d s\right|>\delta\right]=-\infty,
\end{gathered}
$$

and

$$
\limsup _{N \rightarrow \infty} \frac{1}{a_{N}} \log \mathbb{P}_{\rho}^{N}\left[\left|\int_{0}^{t} \rho\left(\rho-\eta_{s}^{M, \mathrm{~L}}(-1)\right) G_{s} d s\right|>\delta\right]=-\infty .
$$

We only prove (2.2) and (2.3), since the remaining two terms are similar.

For any $A>0$, by Chebyshev's inequality, the formula on the left-hand side of (2.2) is bounded from above by

$$
-\frac{A \delta}{a_{N}}+\frac{1}{a_{N}} \log \mathbb{E}_{\rho}^{N}\left[\exp \left\{A\left|\int_{0}^{t}\left(\eta_{s}(0)-\eta_{s}^{M, \mathrm{R}}(0)\right)\left(1-\eta_{s}(-1)\right) G_{s} d s\right|\right\}\right] .
$$

Since $e^{|x|} \leq e^{x}+e^{-x}$, we can remove the modulus in the expectation above. By the FeynmanKac formula (see [14, Lemma A.1.7.2] by Kipnis and Landim for example), the second term in (2.4) is bounded by

$$
\frac{1}{a_{N}} \int_{0}^{t} d s \sup _{f \text { density }}\left\{A G_{s} \int\left(\eta(0)-\eta^{M, \mathrm{R}}(0)\right)(1-\eta(-1)) f(\eta) d \nu_{\rho}-\mathcal{D}_{N}\left(f ; \nu_{\rho}\right)\right\}
$$

where $\mathcal{D}_{N}\left(f ; \nu_{\rho}\right)$ is the Dirichlet form of $f$ associated with $\nu_{\rho}$ given by

$$
\begin{aligned}
\mathcal{D}_{N}\left(f ; \nu_{\rho}\right) & :=\left\langle\sqrt{f},\left(-\mathcal{L}_{N}\right) \sqrt{f}\right\rangle_{\nu_{\rho}} \\
& =N\left[\sqrt{f\left(\eta^{-1,0}\right)}-\sqrt{f(\eta)}\right]^{2}+N^{2} \sum_{\substack{x \in \mathbb{T}_{N}, x \neq-1}}\left[\sqrt{f\left(\eta^{x, x+1}\right)}-\sqrt{f(\eta)}\right]^{2} .
\end{aligned}
$$


We first write $\eta(0)-\eta^{M, \mathrm{R}}(0)$ as a telescope sum,

$$
\eta(0)-\eta^{M, \mathrm{R}}(0)=\frac{1}{M} \sum_{x=0}^{M-1} \sum_{y=0}^{x-1}(\eta(y)-\eta(y+1)) .
$$

Making the transformations $\eta \rightarrow \eta^{y, y+1}$, by Cauchy-Schwartz inequality, we obtain that there exists a constant $C$ only depending on $G$ such that for any $B>0$,

$$
\begin{aligned}
& A G_{s} \int\left(\eta(0)-\eta^{M, \mathrm{R}}(0)\right)(1-\eta(-1)) f(\eta) d \nu_{\rho} \\
& =\frac{A G_{s}}{2 M} \sum_{x=0}^{M-1} \sum_{y=0}^{x-1} \int(\eta(y)-\eta(y+1))(1-\eta(-1))\left(f(\eta)-f\left(\eta^{y, y+1}\right)\right) d \nu_{\rho} \\
& \leq \frac{A B\|G\|_{\infty}}{4 M} \sum_{x=0}^{M-1} \sum_{y=0}^{x-1} \int\left(\sqrt{f(\eta)}-\sqrt{f\left(\eta^{y, y+1}\right)}\right)^{2} d \nu_{\rho} \\
& \quad+\frac{A\|G\|_{\infty}}{4 B M} \sum_{x=0}^{M-1} \sum_{y=0}^{x-1} \int\left(\sqrt{f(\eta)}+\sqrt{f\left(\eta^{y, y+1}\right)}\right)^{2} d \nu_{\rho} \\
& \leq C\left(\frac{A B}{N^{2}} \mathcal{D}_{N}\left(f ; \nu_{\rho}\right)+\frac{A M}{B}\right) .
\end{aligned}
$$

Taking $B=N^{2} A^{-1} C^{-1}$, we bound (2.4) by

$$
\inf _{A>0}\left\{-\frac{A \delta}{a_{N}}+\frac{A^{2} C^{2} t M}{N^{2} a_{N}}\right\}=-\frac{\delta^{2} N^{2}}{4 C^{2} t M a_{N}} .
$$

We prove (2.2) by choosing $M$ such that $M a_{N} \ll N^{2}$.

As above, for any $A>0$, the formula on the left-hand side of (2.3) is bounded by

$$
-\frac{A \delta}{a_{N}}+\frac{1}{a_{N}} \log \mathbb{E}_{\rho}^{N}\left[\exp \left\{A\left|\int_{0}^{t}\left(\eta_{s}^{M, \mathrm{R}}(0)-\rho\right)\left(1-\eta_{s}^{M, \mathrm{~L}}(-1)\right) G_{s} d s\right|\right\}\right] .
$$

As before, we can first remove the modulus. By Jensen's inequality and the invariance of the measure $\nu_{\rho}$, we bound the above formula by

$$
-\frac{A \delta}{a_{N}}+\frac{1}{a_{N}} \log \left(\frac{1}{t} \int_{0}^{t} d s \mathrm{E}_{\nu_{\rho}}\left[\exp \left\{\operatorname{AtG}_{\mathrm{s}}\left(\eta^{\mathrm{M}, \mathrm{R}}(0)-\rho\right)\left(1-\eta^{\mathrm{M}, \mathrm{L}}(-1)\right)\right\}\right]\right) .
$$

By Taylor's expansion, the expectation in the above formula is less than or equal to

$$
\begin{aligned}
& \sum_{k \geq 0} \frac{A^{2 k} t^{2 k}\|G\|_{\infty}^{2 k}}{(2 k) !} \mathrm{E}_{\nu_{\rho}}\left[\left(\eta^{\mathrm{M}, \mathrm{R}}(0)-\rho\right)^{2 \mathrm{k}}\right] \\
& \quad+\sum_{k \geq 0} \frac{A^{2 k+1} t^{2 k+1}\|G\|_{\infty}^{2 k+1}}{(2 k+1) !} \mathrm{E}_{\nu_{\rho}}\left[\left|\eta^{\mathrm{M}, \mathrm{R}}(0)-\rho\right|^{2 \mathrm{k}+1}\right] \\
& \leq\left(1+A t\|G\|_{\infty}\right) \sum_{k \geq 0} \frac{A^{2 k} t^{2 k}\|G\|_{\infty}^{2 k}}{(2 k) !} \mathrm{E}_{\nu_{\rho}}\left[\left(\eta^{\mathrm{M}, \mathrm{R}}(0)-\rho\right)^{2 \mathrm{k}}\right] .
\end{aligned}
$$


Some computations (see the appendix) show that there exists a constant $C(\rho)$ such that

$$
\mathrm{E}_{\nu_{\rho}}\left[\left(\eta^{M, \mathrm{R}}(0)-\rho\right)^{2 k}\right] \leq \frac{C(\rho)^{k} k !}{M^{k}}
$$

Since $2^{k}(k !)^{2} \leq(2 k)$ !, the expectation in $(2.5)$ is bounded by $C A \exp \left\{C A^{2} / M\right\}$ for some constant $C=C(t, G, \rho)$. Therefore, we bound $(2.5)$ by

$$
-\frac{A \delta}{a_{N}}+\frac{C A^{2}}{M a_{N}}+\frac{\log C+\log A}{a_{N}} .
$$

We finish the proof by taking $M=[N / 2]$ and $A=M \delta /(2 C)$.

Lemma 2.2. For any $G \in C([0, T] \times[0,1])$ and any $\delta, t>0$,

$$
\limsup _{N \rightarrow \infty} \frac{1}{a_{N}} \log \mathbb{P}_{\rho}^{N}\left[\left|\int_{0}^{t} \frac{1}{N} \sum_{\substack{x \in \mathbb{T}_{N}, x \neq-1}}\left[\left(\eta_{s}(x)-\eta_{s}(x+1)\right)^{2}-2 \rho(1-\rho)\right] G_{s}\left(\frac{x}{N}\right) d s\right|>\delta\right]=-\infty .
$$

Lemma 2.3. Let $G \in C[0,1]$. Then for any $t>0$,

$$
\limsup _{A \rightarrow \infty} \limsup _{N \rightarrow \infty} \frac{N}{a_{N}^{2}} \log \mathbb{P}_{\rho}^{N}\left[\sup _{0 \leqslant t \leqslant T}\left|\int_{0}^{t}\left\langle\mu_{s}^{N}, G\right\rangle d s\right|>A\right]=-\infty
$$

and for any $\epsilon, t>0$,

$$
\limsup _{\delta \rightarrow 0} \limsup _{N \rightarrow \infty} \frac{N}{a_{N}^{2}} \log \mathbb{P}_{\rho}^{N}\left[\sup _{|t-s| \leq \delta}\left|\int_{s}^{t}\left\langle\mu_{u}^{N}, G\right\rangle d u\right|>\epsilon\right]=-\infty .
$$

The proof of [13, Lemmas 2.1 and 2.2] also applies to the above two lemmas. The main ingredients are the invariance of the Bernoulli product measure $\nu_{\rho}$. For that reason we omit the proof.

\section{Upper Bound}

In this section, we prove (1.6) the moderate deviations upper bound. The strategy is first proving upper bound over compact sets, and then extending to closed sets, which follows from the exponential tightness.

Fix $G \in \mathscr{G}$. By Feynman-Kac formula (see [14, A.1.7]),

$$
M_{t}^{N}(G):=\frac{f\left(t, \eta_{t}\right)}{f\left(0, \eta_{0}\right)} \exp \left\{-\int_{0}^{t} \frac{\partial_{s} f+\mathcal{L}_{N} f}{f}\left(s, \eta_{s}\right) d s\right\}
$$

is a positive mean-one martingale, where

$$
f(t, \eta):=f_{G}(t, \eta):=\exp \left\{\frac{a_{N}}{N} \sum_{x \in \mathbb{T}_{N}}(\eta(x)-\rho) G_{t}(x / N)\right\}
$$


Notice that

$$
f\left(t, \eta_{t}\right)=\exp \left\{\frac{a_{N}^{2}}{N}\left\langle\mu_{t}^{N}, G_{t}\right\rangle\right\} .
$$

A simple calculation yields that

$$
\begin{aligned}
\left(\partial_{s} f+\mathcal{L}_{N} f\right)\left(s, \eta_{s}\right) & =f\left(s, \eta_{s}\right)\left(\frac{a_{N}^{2}}{N}\left\langle\mu_{s}^{N}, \partial_{s} G_{s}\right\rangle\right. \\
& +N\left[\exp \left\{\frac{a_{N}}{N}\left(\eta_{s}(-1)-\eta_{s}(0)\right)\left(G_{s}\left(\frac{0}{N}\right)-G_{s}\left(\frac{-1}{N}\right)\right)\right\}-1\right] \\
& \left.+\sum_{\substack{x \in \mathbb{T}_{N}, x \neq-1}} N^{2}\left[\exp \left\{\frac{a_{N}}{N}\left(\eta_{s}(x)-\eta_{s}(x+1)\right)\left(G_{s}\left(\frac{x+1}{N}\right)-G_{s}\left(\frac{x}{N}\right)\right)\right\}-1\right]\right) .
\end{aligned}
$$

By Taylor's expansion,

$$
\begin{aligned}
& N\left[\exp \left\{\frac{a_{N}}{N}\left(\eta_{s}(-1)-\eta_{s}(0)\right)\left(G_{s}\left(\frac{0}{N}\right)-G_{s}\left(\frac{-1}{N}\right)\right)\right\}-1\right] \\
& =a_{N}\left(\eta_{s}(-1)-\eta_{s}(0)\right)\left(G_{s}\left(\frac{0}{N}\right)-G_{s}\left(\frac{-1}{N}\right)\right) \\
& +\frac{a_{N}^{2}}{2 N}\left(\eta_{s}(-1)-\eta_{s}(0)\right)^{2}\left(G_{s}\left(\frac{0}{N}\right)-G_{s}\left(\frac{-1}{N}\right)\right)^{2}+\mathcal{O}_{G}\left(\frac{a_{N}^{3}}{N^{2}}\right),
\end{aligned}
$$

and for $x \neq-1$,

$$
\begin{aligned}
& N^{2}\left[\exp \left\{\frac{a_{N}}{N}\left(\eta_{s}(x)-\eta_{s}(x+1)\right)\left(G_{s}\left(\frac{x+1}{N}\right)-G_{s}\left(\frac{x}{N}\right)\right)\right\}-1\right] \\
& =N a_{N}\left(\eta_{s}(x)-\eta_{s}(x+1)\right)\left(G_{s}\left(\frac{x+1}{N}\right)-G_{s}\left(\frac{x}{N}\right)\right) \\
& +\frac{a_{N}^{2}}{2}\left(\eta_{s}(x)-\eta_{s}(x+1)\right)^{2}\left(G_{s}\left(\frac{x+1}{N}\right)-G_{s}\left(\frac{x}{N}\right)^{2}\right)+\mathcal{O}_{G}\left(\frac{a_{N}^{3}}{N^{4}}\right) .
\end{aligned}
$$

Using the summation by parts formula,

$$
\begin{aligned}
M_{t}^{N}(G) & =\exp \frac{a_{N}^{2}}{N}\left\{\left\langle\mu_{t}^{N}, G_{t}\right\rangle-\left\langle\mu_{0}^{N}, G_{0}\right\rangle-\int_{0}^{t}\left\langle\mu_{s}^{N},\left(\partial_{s}+\tilde{\Delta}\right) G_{s}\right\rangle d s\right. \\
& -\int_{0}^{t} \frac{N}{a_{N}}\left(\eta_{s}(-1)-\eta_{s}(0)\right)\left(G_{s}\left(\frac{0}{N}\right)-G_{s}\left(\frac{-1}{N}\right)\right) d s \\
& -\int_{0}^{t} \frac{1}{2}\left(\eta_{s}(-1)-\eta_{s}(0)\right)^{2}\left(G_{s}\left(\frac{0}{N}\right)-G_{s}\left(\frac{-1}{N}\right)\right)^{2} d s \\
& -\int_{0}^{t} \frac{N}{a_{N}}\left(\left(\eta_{s}(0)-\rho\right) \nabla_{N} G_{s}\left(\frac{0}{N}\right)-\left(\eta_{s}(-1)-\rho\right) \nabla_{N} G_{s}\left(\frac{-2}{N}\right)\right) d s \\
& -\int_{0}^{t} \frac{1}{2 N} \sum_{\substack{x \in \mathbb{T}_{N} \\
x \neq-1}}\left(\eta_{s}(x)-\eta_{s}(x+1)\right)^{2}\left(\nabla_{N} G_{s}\left(\frac{x}{N}\right)\right)^{2} d s \\
& \left.+\mathcal{O}_{G}\left(\frac{a_{N}}{N}\right)+\mathcal{O}_{G}\left(\frac{1}{a_{N}}\right)\right\},
\end{aligned}
$$


where $\nabla_{N}$ is the discrete space derivative, $\nabla_{N} G_{s}(x / N):=N\left[G_{s}((x+1) / N)-G_{s}(x / N)\right]$. By the boundary condition (1.3) imposed on $G$, the sum of (3.3) and (3.5) is of order $\mathcal{O}_{G}\left(a_{N}^{-1}\right)$. Therefore,

$$
\begin{aligned}
M_{t}^{N}(G) & =\exp \frac{a_{N}^{2}}{N}\left\{\ell_{t}\left(\mu^{N}\right)-\int_{0}^{t} \frac{1}{2}\left(\eta_{s}(-1)-\eta_{s}(0)\right)^{2}\left(G_{s}\left(\frac{0}{N}\right)-G_{s}\left(\frac{-1}{N}\right)\right)^{2} d s\right. \\
& \left.-\int_{0}^{t} \frac{1}{2 N} \sum_{\substack{x \in \mathbb{T}_{N}, x \neq-1}}\left(\eta_{s}(x)-\eta_{s}(x+1)\right)^{2}\left(\nabla_{N} G_{s}\left(\frac{x}{N}\right)\right)^{2} d s+\mathcal{O}_{G}\left(\frac{1}{a_{N}}\right)\right\} .
\end{aligned}
$$

Lemma 3.1 (Upper bounds over compact sets). For any compact set $K \subset D([0, T], \mathscr{M})$,

$$
\limsup _{N \rightarrow \infty} \frac{N}{a_{N}^{2}} \log Q_{\rho}^{N}[K] \leq-\inf _{\mu \in K} I(\mu) .
$$

Proof. For any $\delta>0$ and any $G \in \mathscr{G}$, let

$$
\begin{aligned}
& B_{N, \delta}=\left\{\left|\int_{0}^{T} \sum_{\substack{x \in \mathbb{T}_{N}, x \neq-1}} \frac{1}{2 N}\left(\eta_{t}(x)-\eta_{t}(x+1)\right)^{2}\left(\nabla_{N} G_{t}\left(\frac{x}{N}\right)\right)^{2} d t-\int_{0}^{T} \int_{0}^{1} \rho(1-\rho)\left(\nabla G_{t}(u)\right)^{2} d t d u\right|<\delta\right\} \\
& \bigcap\left\{\left|\int_{0}^{T}\left[\frac{1}{2}\left(\eta_{t}(-1)-\eta_{t}(0)\right)^{2}-\rho(1-\rho)\right]\left(G_{t}\left(\frac{0}{N}\right)-G_{t}\left(\frac{-1}{N}\right)\right)^{2} d t\right|<\delta\right\} .
\end{aligned}
$$

By Lemmas 2.1,2.2 and the assumption $a_{N} \ll N$,

$$
\limsup _{N \rightarrow \infty} \frac{N}{a_{N}^{2}} \log \mathbb{P}_{\rho}^{N}\left[B_{N, \delta}^{c}\right]=-\infty
$$

Therefore, for any $\gamma \in \mathscr{G}_{0}$,

$$
\begin{aligned}
\limsup _{N \rightarrow \infty} \frac{N}{a_{N}^{2}} \log \mathbb{P}_{\rho}^{N}\left[\mu^{N} \in K\right] \leq & \limsup _{N \rightarrow \infty} \frac{N}{a_{N}^{2}} \log \mathbb{P}_{\rho}^{N}\left[\left\{\mu^{N} \in K\right\} \cap B_{N, \delta}\right] \\
& =\limsup _{N \rightarrow \infty} \frac{N}{a_{N}^{2}} \log \mathbb{E}_{\rho}^{N}\left[\left(M_{T}^{N}(G)\right)^{-1} M_{T}^{N}(G) \mathbf{1}_{\left.\left\{\mu^{N} \in K\right\} \cap B_{N, \delta}\right]}\right. \\
\leq & \sup _{\mu \in K}\left\{-\ell_{T}(\mu)+\rho(1-\rho) \int_{0}^{T}\left(G_{t}(0)-G_{t}(1)\right)^{2} d t\right. \\
& \left.+\rho(1-\rho) \int_{0}^{T} \int_{0}^{1}\left(\partial_{u} G_{t}(u)\right)^{2} d u d t-\mu_{0}(\gamma)\right\}+\mathcal{O}(\delta) \\
& +\limsup _{N \rightarrow \infty} \frac{N}{a_{N}^{2}} \log \mathbb{E}_{\rho}^{N}\left[M_{T}^{N}(G) \exp \left\{\frac{a_{N}^{2}}{N}\left\langle\mu_{0}^{N}, \gamma\right\rangle\right\}\right] .
\end{aligned}
$$

Because $\left\{M_{t}^{N}(G)\right\}$ is a mean one martingale and $\nu_{\rho}$ is a product measure, direct calculations yield that

$$
\limsup _{N \rightarrow \infty} \frac{N}{a_{N}^{2}} \log \mathbb{E}_{\rho}^{N}\left[M_{T}^{N}(G) \exp \left\{\frac{a_{N}^{2}}{N}\left\langle\mu_{0}^{N}, \gamma\right\rangle\right\}\right]=\frac{\rho(1-\rho)}{2} \int_{0}^{1} \gamma(u)^{2} d u
$$


Letting $\delta \rightarrow 0$, and then minimizing over $G \in \mathscr{G}, \gamma \in \mathscr{G}_{0}$,

$$
\begin{gathered}
\limsup _{N \rightarrow \infty} \frac{N}{a_{N}^{2}} \log \mathbb{P}_{\rho}^{N}\left[\mu^{N} \in K\right] \leq \inf _{\substack{G \in \mathscr{G} \\
\gamma \in \mathscr{G}_{0},}} \sup _{\mu \in K}\left\{-\ell_{T}(\mu)+\rho(1-\rho) \int_{0}^{T}\left(G_{t}(0)-G_{t}(1)\right)^{2} d t\right. \\
\left.\quad+\rho(1-\rho) \int_{0}^{T} \int_{0}^{1}\left(\partial_{u} G_{t}(u)\right)^{2} d u d t-\mu_{0}(\gamma)+\frac{\rho(1-\rho)}{2} \int_{0}^{1} \gamma(u)^{2} d u\right\} .
\end{gathered}
$$

In order to exchange the supremum and infimum above, we use the following version of Minimax Theorem proved by Nikaidô.

Theorem 3.2 (Minimax Theorem, [15, Theorem 1]). Let $\mathbf{X}$ be a linear space endowed with separative topology and $\mathbf{Y}$ a linear space. Moreover, assume $\mathbf{X}$ is compact. Let $f: \mathbf{X} \times \mathbf{Y} \rightarrow \mathbb{R}$ satisfy that $f(x, y)$ is convex in $y$ for each fixed $x$, and concave in $x$ for each fixed $y$. Furthermore, $f(x, y)$ is continuous in $x$ for each fixed $y$. If $\sup _{x \in \mathbf{X}} \inf _{y \in \mathbf{Y}} f(x, y)$ is finite, then

$$
\sup _{x \in \mathbf{X}} \inf _{y \in \mathbf{Y}} f(x, y)=\inf _{y \in \mathbf{Y}} \sup _{x \in \mathbf{X}} f(x, y) .
$$

We finish the proof by taking $\mathbf{X}=K \subset D([0, T], \mathscr{M}), \mathbf{Y}=\mathscr{G} \times \mathscr{G}_{0}$ and

$$
\begin{aligned}
f(\mu,(G, \gamma)) & =-\ell_{T}(\mu)+\rho(1-\rho) \int_{0}^{T}\left(G_{t}(0)-G_{t}(1)\right)^{2} d t \\
& +\rho(1-\rho) \int_{0}^{T} \int_{0}^{1}\left(\partial_{u} G_{t}(u)\right)^{2} d u d t-\mu_{0}(\gamma)+\frac{\rho(1-\rho)}{2} \int_{0}^{1} \gamma(u)^{2} d u
\end{aligned}
$$

for any $\mu \in \mathbf{X}$ and $(G, \gamma) \in \mathbf{Y}$.

To extend the moderate deviations upper bound to any closed set, it suffices to show the exponential tightness of the sequence $\left\{Q_{N}\right\}_{N \geq 1}$, which follows from the following Lemma as in [13].

Lemma 3.3. For any $G \in \mathscr{G}_{0}$,

$$
\limsup _{A \rightarrow \infty} \limsup _{N \rightarrow \infty} \frac{N}{a_{N}^{2}} \log \mathbb{P}_{\rho}^{N}\left(\sup _{0 \leqslant t \leqslant T}\left|\left\langle\mu_{t}^{N}, G\right\rangle\right|>A\right)=-\infty
$$

and for any $\epsilon>0$,

$$
\limsup _{\delta \rightarrow 0} \limsup _{N \rightarrow \infty} \frac{N}{a_{N}^{2}} \log \mathbb{P}_{\rho}^{N}\left(\sup _{0<t \leqslant \delta}\left|\left\langle\mu_{t}^{N}-\mu_{0}^{N}, G\right\rangle\right|>\varepsilon\right)=-\infty .
$$

We first explain why the above lemma implies exponential tightness. For any $m \in \mathbb{N}, k \in \mathbb{Z}$ and any $\delta, A>0$, define

$$
B_{k, A}=\left\{\sup _{0 \leq t \leq T}\left|\mu_{t}\left(\theta_{k}\right)\right| \leq A\right\}, \quad B_{k, m, \delta}=\left\{\sup _{0 \leq|t-s| \leq \delta}\left|\left(\mu_{t}-\mu_{s}\right)\left(\theta_{k}\right)\right| \leq \frac{1}{m}\right\} .
$$

Then by Lemma 3.3, for any $n>0$, there exist $A=A(n, k)$ and $\delta=\delta(m, k, n)$ such that

$$
\sup _{N \geq 1} Q_{\rho}^{N}\left[B_{k, A}^{c}\right]<e^{-\left(a_{N}^{2} / N\right) n k}, \quad \sup _{N \geq 1} Q_{\rho}^{N}\left[B_{k, m, \delta}^{c}\right]<e^{-\left(a_{N}^{2} / N\right) n k m} .
$$


Let

$$
\mathcal{K}_{n}=\left\{\bigcap_{k \geq 1} B_{k, A(n, k)}\right\} \bigcap\left\{\bigcap_{k, m \geq 1} B_{k, m, \delta(m, k, n)}\right\} .
$$

It can be checked that $\mathcal{K}_{n}$ is a compact set for each $n \geq 1$. Moreover, $Q_{\rho}^{N}\left[\mathcal{K}_{n}^{c}\right]$ is bounded by a multiple of $\exp \left\{-\left(a_{N}^{2} / N\right) n\right\}$. This proves the exponential tightness.

Proof of Lemma 3.3. We first prove (3.9). Since (3.4) and (3.6) are bounded, we only need to show that

$$
\limsup _{A \rightarrow \infty} \limsup _{N \rightarrow \infty} \frac{N}{a_{N}^{2}} \log \mathbb{P}_{\rho}^{N}\left[\sup _{0 \leqslant t \leqslant T}\left|\frac{N}{a_{N}^{2}} \log M_{t}^{N}(G)+\left\langle\mu_{0}^{N}, G\right\rangle+\int_{0}^{t}\left\langle\mu_{s}^{N}, \tilde{\Delta} G\right\rangle d s\right|>A\right]=-\infty,
$$

which is a consequence of

$$
\begin{array}{r}
\limsup _{A \rightarrow \infty} \limsup _{N \rightarrow \infty} \frac{N}{a_{N}^{2}} \log \mathbb{P}_{\rho}^{N}\left[\sup _{0 \leqslant t \leqslant T}\left|\frac{N}{a_{N}^{2}} \log M_{t}^{N}(G)\right|>A / 3\right]=-\infty, \\
\limsup _{A \rightarrow \infty} \limsup _{N \rightarrow \infty} \frac{N}{a_{N}^{2}} \log \mathbb{P}_{\rho}^{N}\left[\frac{1}{a_{N}}\left|\sum_{x \in \mathbb{T}_{N}}\left(\eta_{0}(x)-\rho\right) G(x / N)\right|>A / 3\right]=-\infty,
\end{array}
$$

and

$$
\limsup _{A \rightarrow \infty} \limsup _{N \rightarrow \infty} \frac{N}{a_{N}^{2}} \log \mathbb{P}_{\rho}^{N}\left[\sup _{0 \leqslant t \leqslant T}\left|\int_{0}^{t}\left\langle\mu_{s}^{N}, \tilde{\Delta} G\right\rangle d s\right|>A / 3\right]=-\infty .
$$

Notice that (3.13) follows from Lemma 2.3. To prove (3.11), without loss of generality, we first remove the modulus since otherwise we can replace $G$ by $-G$. Then

$$
\begin{aligned}
& \mathbb{P}_{\rho}^{N}\left[\sup _{0 \leqslant t \leqslant T} \frac{N}{a_{N}^{2}} \log M_{t}^{N}(G)>A / 3\right]=\mathbb{P}_{\rho}^{N}\left[\sup _{0 \leqslant t \leqslant T} M_{t}^{N}(G)>\exp \left\{\frac{A a_{N}^{2}}{3 N}\right\}\right] \\
& \leq 4 \exp \left\{-\frac{A a_{N}^{2}}{3 N}\right\} \mathbb{E}_{\rho}^{N}\left[\left(M_{T}^{N}(G)\right)^{2}\right] \leq 4 \exp \left\{C\left(\|G\|_{\infty}^{2}+\left\|G^{\prime}\right\|_{\infty}^{2}\right) T-\frac{A a_{N}^{2}}{3 N}\right\} .
\end{aligned}
$$

This proves (3.11). For (3.12), removing the modulus inside the probability as before and then by Chebyshev's inequality,

$$
\begin{aligned}
& \frac{N}{a_{N}^{2}} \log \mathbb{P}_{\rho}^{N}\left[\frac{1}{a_{N}} \sum_{x \in \mathbb{T}_{N}}\left(\eta_{0}(x)-\rho\right) G(x / N)>A / 3\right] \\
& \leq-A / 3+\frac{N}{a_{N}^{2}} \log \mathbb{E}_{\rho}^{N}\left[\exp \left\{\frac{a_{N}}{N} \sum_{x \in \mathbb{T}_{N}}\left(\eta_{0}(x)-\rho\right) G(x / N)\right\}\right] \\
& =-A / 3+\frac{N}{a_{N}^{2}} \sum_{x \in \mathbb{T}_{N}} \log \left(1+\frac{C(\rho) a_{N}^{2}}{N^{2}} G(x / N)^{2}+\mathcal{O}_{G}\left(\frac{a_{N}^{3}}{N^{3}}\right)\right) \\
& \leq-A / 3+\frac{C(\rho)}{N} \sum_{x \in \mathbb{T}_{N}} G(x / N)^{2}+\mathcal{O}_{G}\left(\frac{a_{N}}{N}\right) .
\end{aligned}
$$


This proves (3.12) by letting $N \rightarrow \infty$ and then $A \rightarrow \infty$.

Next we prove (3.10). Fix $A>0$, which will converge to infinity after $\delta \rightarrow 0, N \rightarrow \infty$. From Equations (3.2)-(3.7) with $G$ replaced by $A G$, we only need to prove (3.10) for the following four terms:

$$
\begin{gathered}
\frac{N}{A a_{N}^{2}} \log M_{t}^{N}(A G), \quad \int_{0}^{t}\left\langle\mu_{s}^{N}, \tilde{\Delta} G\right\rangle d s \\
A \int_{0}^{t} \frac{1}{2}\left(\eta_{s}(-1)-\eta_{s}(0)\right)^{2}\left(G\left(\frac{0}{N}\right)-G\left(\frac{-1}{N}\right)\right)^{2} d s
\end{gathered}
$$

and

$$
A \int_{0}^{t} \frac{1}{2 N} \sum_{\substack{x \in \mathbb{T}_{N}, x \neq-1}}\left(\eta_{s}(x)-\eta_{s}(x+1)\right)^{2}\left(\nabla_{N} G\left(\frac{x}{N}\right)\right)^{2} d s .
$$

Notice that the proof of (3.11) also applies to the martingale term. The second one follows from Lemma 2.3. For the last two terms, notice that they are both bounded by $C(G) \delta A$. The proof is complete.

\section{Lower bound}

For $f, g \in \mathscr{G}_{0}$, we define

$$
\langle f \mid g\rangle=2 \rho(1-\rho)\left[(f(0)-f(1))(g(0)-g(1))+\int_{0}^{1} \partial_{u} f(u) \partial_{u} g(u) d u\right] .
$$

For $f, g \in \mathscr{G}$ and $0 \leq t \leq T$, we define

$$
\langle\langle f, g\rangle\rangle_{t}=\int_{0}^{t}\left\langle f_{s} \mid g_{s}\right\rangle d s .
$$

For simplicity, we write $\langle\langle f, g\rangle\rangle_{T}$ as $\langle\langle f, g\rangle\rangle$. To make $\langle\langle\cdot, \cdot\rangle\rangle$ an inner product, we write $f \simeq g$ if and only if $\langle\langle f-g, f-g\rangle\rangle=0$ and then define $\mathscr{H}$ as the Hilbert space which is the completion of $\mathscr{G} / \simeq$.

For locally square integrable martingales $\left\{M_{t}\right\}_{t \geq 0}$ and $\left\{N_{t}\right\}_{t \geq 0}$, we use $\left\{\langle M, N\rangle_{t}\right\}_{t \geq 0}$ to denote the predictable quadratic-covariation process which is continuous and use $\left\{[M, N]_{t}\right\}_{t \geq 0}$ to denote the optional quadratic-covariation process which satisfies

$$
[M, N]_{T}=\lim _{\sup \left(t_{i+1}-t_{i}\right) \rightarrow 0} \sum_{i}\left(M_{t_{i+1}}-M_{t_{i}}\right)\left(N_{t_{i+1}}-N_{t_{i}}\right) \quad \text { in } L^{2}
$$

where the limit is over all partitions $\left\{t_{i}\right\}$ of $[0, T]$. Note that $[M, N]=\langle M, N\rangle$ when $M$ and $N$ are continuous. For any $H \in C^{1,2}\left([0, T] \times\{0,1\}^{\mathbb{T}_{N}}\right)$, by Dynkin's martingale formula,

$$
\Lambda_{t}^{N}(H):=H\left(t, \eta_{t}\right)-H\left(0, \eta_{0}\right)-\int_{0}^{t}\left(\mathcal{L}_{N}+\partial_{s}\right) H\left(s, \eta_{s}\right) d s
$$

is a martingale and for any $H_{1}, H_{2} \in C^{1,2}\left([0, T] \times\{0,1\}^{\mathbb{T}_{N}}\right)$,

$$
\left\langle\Lambda^{N}\left(H_{1}\right), \Lambda^{N}\left(H_{2}\right)\right\rangle_{t}=\int_{0}^{t} \mathcal{L}_{N}\left(H_{1} H_{2}\right)-H_{1} \mathcal{L}_{N} H_{2}-H_{2} \mathcal{L}_{N} H_{1} d s
$$

The following lemma gives clear expressions of $I_{d y n}$ and $I_{i n i}$. 
Lemma 4.1. (i) If $I_{d y n}(\mu)<+\infty$, then there exists $\psi \in \mathscr{H}$ such that $\ell_{T}(\mu, G)=\langle\langle G, \psi\rangle\rangle$ for any $G \in \mathscr{G}$ and $I_{\text {dyn }}(\mu)=\frac{1}{2}\langle\langle\psi, \psi\rangle\rangle$.

(ii) If $I_{\text {ini }}(\nu)<+\infty$ for $\nu \in \mathscr{M}$, then there exists $\phi \in L^{2}[0,1]$ such that $\nu(G)=\langle\phi$, G $\rangle$ for any $G \in \mathscr{G}_{0}$ and

$$
I_{i n i}(\nu)=\frac{\int_{0}^{1} \phi^{2}(u) d u}{2 \rho(1-\rho)} .
$$

Proof. The proofs of the two parts follow the same strategy, hence we only give the proof of $(i)$. According to the definition of $I_{d y n}$,

$$
I_{d y n}(\mu)=\sup _{G \in \mathscr{G}}\left\{\ell_{T}(\mu, G)-(1 / 2)\langle\langle G, G\rangle\rangle\right\}
$$

If $\ell_{T}(\mu, G) \neq 0$ for some $G$ such that $\langle\langle G, G\rangle\rangle=0$, then

$$
I_{d y n}(\mu) \geq \sup _{c \in \mathbb{R}}\left\{\ell_{T}(\mu, c G)-\frac{1}{2}\langle\langle c G, c G\rangle\rangle\right\}=\sup _{c \in \mathbb{R}}\left\{c \ell_{T}(\mu, G)\right\}=+\infty,
$$

which is contradictory. Therefore, $\ell_{T}(\mu, \cdot)$ is well defined on $\mathscr{G} / \simeq$. For $G \in \mathscr{G} / \simeq$ such that $G \neq 0, \ell_{T}(\mu, c G)-(1 / 2)\langle\langle c G, c G\rangle\rangle$ obtains maximum $\frac{\ell_{T}^{2}(\mu, G)}{2\langle\langle G, G\rangle\rangle}$ at $c=\frac{\ell_{T}(\mu, G)}{\langle\langle G, G\rangle\rangle}$. Therefore,

$$
I_{d y n}(\mu)=\sup _{G \in \mathscr{G} / \simeq, G \neq 0} \frac{\ell_{T}^{2}(\mu, G)}{2\langle\langle G, G\rangle\rangle} .
$$

Since $I_{d y n}(\mu)<+\infty, \ell_{T}(\mu, \cdot)$ can be extended to a bounded linear function on $\mathscr{H}$. As a result, the existence of $\psi$ follows from Riesz's representation theorem and $I_{d y n}(\mu)=\frac{1}{2}\langle\langle\psi, \psi\rangle\rangle$ follows from Cauchy-Schwartz's inequality.

For $\phi \in \mathscr{G}_{0}$ and sufficiently large $N$, denote by $\nu_{N, \phi}$ the product measure on $\{0,1\}^{\mathbb{T}_{N}}$ with marginals given by

$$
\nu_{N, \phi}\{\eta: \eta(x)=1\}=\rho+\frac{a_{N}}{N} \phi\left(\frac{x}{N}\right), \quad x \in \mathbb{T}_{N},
$$

and by $\mathbb{P}_{\phi}^{N}$ the law of the process $\left\{\eta_{t}\right\}_{t \geq 0}$ with initial distribution $\nu_{N, \phi}$. For any $G \in \mathscr{G}$, denote by $\widehat{\mathbb{P}}_{\phi, G}^{N}$ the probability measure on $D\left([0, T],\{0,1\}^{\mathbb{T}_{N}}\right)$ such that

$$
\frac{d \widehat{\mathbb{P}}_{\phi, G}^{N}}{d \mathbb{P}_{\phi}^{N}}=M_{T}^{N}(G)
$$

Lemma 4.2. For any $G \in \mathscr{G}$ and any $\phi \in \mathscr{G}_{0},\left\{\mu_{t}^{N}\right\}_{0 \leq t \leq T}$ converges in $\widehat{\mathbb{P}}_{\phi, G}^{N}$-probability to $\mu^{G}=\left\{\mu_{t}^{G}\right\}_{0 \leq t \leq T}$ as $N \rightarrow \infty$, where $\mu^{G}$ is the unique element in $D([0, T], \mathscr{M})$ such that

$$
\left\{\begin{array}{l}
\frac{d}{d t} \mu_{t}^{G}(h)=\mu_{t}^{G}(\tilde{\Delta} h)+\left\langle h \mid G_{t}\right\rangle \\
\mu_{0}^{G}(h)=\int_{0}^{1} \phi(u) h(u) d u
\end{array}\right.
$$

for any $h \in \mathscr{G}_{0}$ and $0 \leq t \leq T$. 
Remark 4.3. Intuitively but not rigorously, integrating by parts, $\mu_{t}^{G}$ given by (4.3) should be a signed measure such that $\mu_{t}^{G}(d u)=\rho(t, u) d u$, where $\rho(t, u)$ is the solution to the PDE

$$
\left\{\begin{array}{l}
\partial_{t} \rho(t, u)=\tilde{\Delta} \rho(t, u)-2 \rho(1-\rho) \tilde{\Delta} G_{t}(u) \\
\rho(0, u)=\phi(u), 0 \leq u \leq 1 \\
\rho(t, \cdot) \in \mathscr{G}_{0}, \quad 0 \leq t \leq T
\end{array}\right.
$$

However, as we have discussed in Remark 1.2, we do not manage to prove the uniqueness or existence to this PDE. That's why we only consider $\mu^{G}$ as the solution to an equation on $\mathscr{M}$ the space of linear functionals on $\mathscr{G}_{0}$, the uniqueness and existence of which we can show rigorously.

To prove Lemma 4.2, we need some preparation. For $h \in \mathscr{G}$, we write

$$
F_{h}(t, \eta)=\frac{1}{a_{N}} \sum_{x \in \mathbb{T}_{N}}(\eta(x)-\rho) h_{t}\left(\frac{x}{N}\right)
$$

and hence

$$
\Lambda_{t}^{N}\left(F_{h}\right)=\left\langle\mu_{t}^{N}, h_{t}\right\rangle-\left\langle\mu_{0}^{N}, h_{0}\right\rangle-\int_{0}^{t}\left(\mathcal{L}_{N}+\partial_{s}\right)\left\langle\mu_{s}^{N}, h_{s}\right\rangle d s
$$

Lemma 4.4. For any $\phi \in \mathscr{G}_{0}, G, h \in \mathscr{G}$,

$$
\left[\Lambda^{N}\left(F_{h}\right), \Lambda^{N}\left(F_{h}\right)\right]_{T}=o_{\exp }\left(a_{N}^{2}\right)
$$

under both $\mathbb{P}_{\phi}^{N}$ and $\widehat{\mathbb{P}}_{\phi, G}^{N}$.

Proof. We first show that Equation (4.4) holds under $\mathbb{P}_{\phi}^{N}$. According to the definition of $\Lambda_{t}^{N}\left(F_{h}\right)$,

$$
\left[\Lambda^{N}\left(F_{h}\right), \Lambda^{N}\left(F_{h}\right)\right]_{T}=\sum_{0 \leq s \leq T}\left[\left\langle\mu_{s}^{N}, h_{s}\right\rangle-\left\langle\mu_{s-}^{N}, h_{s-}\right\rangle\right]^{2}
$$

Recall that $\left\{Y_{i}(\cdot)\right\}_{i \in \mathbb{T}_{N}}$ are independent Poisson processes. If $s$ is an event moment of $Y_{i}(\cdot)$, then

$$
\left\langle\mu_{s}^{N}, h_{s}\right\rangle-\left\langle\mu_{s-}^{N}, h_{s-}\right\rangle=\frac{1}{a_{N}}\left(h_{s-}\left(\frac{i+1}{N}\right)-h_{s-}\left(\frac{i}{N}\right)\right)\left(\eta_{s-}(i)-\eta_{s-}(i+1)\right) .
$$

Consequently, let $C_{h}=\sup _{\substack{0 \leq t \leq T, 0 \leq u \leq 1}}|h(t, u)|$ and $D_{h}=\sup _{\substack{0 \leq t \leq T, 0 \leq u \leq 1}}\left|\partial_{u} h(t, u)\right|$, then

$$
\left[\Lambda^{N}\left(F_{h}\right), \Lambda^{N}\left(F_{h}\right)\right]_{T} \leq \frac{4}{a_{N}^{2}} \sum_{i \neq-1} \frac{Y_{i}(T)}{N^{2}} D_{h}^{2}+\frac{16}{a_{N}^{2}} C_{h}^{2} Y_{-1}(T)
$$

according to Lagrange's mean value theorem and the fact that there is at most one particle per site. By Chebyshev's inequality, for any $\theta>0$,

$$
\begin{aligned}
& \mathbb{P}_{\phi}^{N}\left(\left[\Lambda^{N}\left(F_{h}\right), \Lambda^{N}\left(F_{h}\right)\right]_{T} \geq \epsilon\right) \leq \exp \left\{-\theta a_{N}^{2} \epsilon\right\} E\left[\exp \left\{4 \theta \sum_{i \neq-1} \frac{Y_{i}(T)}{N^{2}} D_{h}^{2}+16 \theta C_{h}^{2} Y_{-1}(T)\right\}\right] \\
& =\exp \left\{-\theta a_{N}^{2} \epsilon\right\}\left[\exp \left\{N^{2} T\left(\exp \left\{\frac{4 \theta D_{h}^{2}}{N^{2}}\right\}-1\right)\right\}\right]^{N-1} \exp \left\{N T\left(\exp \left\{16 \theta C_{h}^{2}\right\}-1\right)\right\} .
\end{aligned}
$$


Then

$$
\limsup _{N \rightarrow+\infty} \frac{1}{a_{N}^{2}} \log \mathbb{P}_{\phi}^{N}\left(\left[\Lambda^{N}\left(F_{h}\right), \Lambda^{N}\left(F_{h}\right)\right]_{T} \geq \epsilon\right) \leq-\theta \epsilon .
$$

This proves Equation (4.4) under $\mathbb{P}_{\phi}^{N}$ since $\theta$ is arbitrary.

Now we only need to show that Equation (4.4) holds under $\widehat{\mathbb{P}}_{\phi, G}^{N}$. According to the definition of $\widehat{\mathbb{P}}_{\phi, G}^{N}$ and Cauchy-Schwartz inequality, for any $\epsilon>0$,

$$
\widehat{\mathbb{P}}_{\phi, G}^{N}\left(\left[\Lambda^{N}\left(F_{h}\right), \Lambda^{N}\left(F_{h}\right)\right]_{T} \geq \epsilon\right) \leq \sqrt{\mathbb{P}_{\phi}^{N}\left(\left[\Lambda^{N}\left(F_{h}\right), \Lambda^{N}\left(F_{h}\right)\right]_{T} \geq \epsilon\right)} \sqrt{\mathbb{E}_{\phi}^{N}\left[\left(M_{T}^{N}(G)\right)^{2}\right]}
$$

Recall the expressions of $M_{t}^{N}(G)$ given in (3.2)-(3.7). It is not difficult to check that there exists a finite constant $C$ independent of $N$ such that $M_{T}^{N}(G) \leq e^{C a_{N}}$ for sufficiently large $N$. Therefore, Equation (4.4) also holds under $\widehat{\mathbb{P}}_{\phi, G}^{N}$.

Proof of Lemma 4.2. The existence and uniqueness of Equation (4.3) are given in the appendix. It remains to show that $\mu^{N}$ converges weakly under $\widehat{\mathbb{P}}_{\phi, G}^{N}$ to this unique solution $\mu^{G}$ as $N \rightarrow \infty$. To achieve this purpose, we need to investigate the martingale $\left\{M_{t}^{N}(G)\right\}_{t \geq 0}$ in (3.1) and to utilize a generalized version of Girsanov's theorem introduced in [16] by Schuppen and Wong.

Recall the definition of $\Lambda_{t}^{N}(f)$ in (4.1) and that for any $G \in \mathscr{G}$,

$$
f_{G}(t, \eta)=\exp \left\{\frac{a_{N}}{N} \sum_{i \in \mathbb{T}_{N}}(\eta(i)-\rho) G_{t}\left(\frac{i}{N}\right)\right\} .
$$

According to Ito's formula,

$$
d M_{t}^{N}(G)=f_{G}\left(0, \eta_{0}\right)^{-1} \exp \left\{-\int_{0}^{t} \frac{\left(\partial_{s}+\mathcal{L}_{N}\right) f_{G}}{f_{G}}\left(s, \eta_{s}\right) d s\right\} d \Lambda_{t}^{N}\left(f_{G}\right) .
$$

For any $t \geq 0$, let

$$
\widetilde{\Lambda}_{t}^{N}\left(f_{G}\right)=\int_{0}^{t} \frac{1}{f_{G}\left(s-, \eta_{s-}\right)} d \Lambda_{s}^{N}\left(f_{G}\right)
$$

then

$$
d M_{t}^{N}(G)=M_{t-}^{N}(G) d \widetilde{\Lambda}_{t}^{N}\left(f_{G}\right) .
$$

For any local martingale $\left\{M_{t}\right\}_{t \geq 0}$ under $\mathbb{P}_{\phi}^{N}$, let

$$
\widehat{M}_{t}=M_{t}-\left\langle M, \widetilde{\Lambda}^{N}\left(f_{G}\right)\right\rangle_{t}
$$

By Equation (4.5) and the generalized version of Girsanov's theorem [16], $\left\{\widehat{M}_{t}\right\}_{t \geq 0}$ is a local martingale under $\widehat{\mathbb{P}}_{\phi, G}^{N}$ and $[\widehat{M}, \widehat{M}]=[M, M]$ under both $\mathbb{P}_{\phi}^{N}$ and $\widehat{\mathbb{P}}_{\phi, G}^{N}$. Therefore, for any $h \in \mathscr{G}$,

$$
\left.\left\langle\mu_{t}^{N}, h_{t}\right\rangle=\left\langle\mu_{0}^{N}, h_{0}\right\rangle+\int_{0}^{t}\left(\partial_{s}+\mathcal{L}_{N}\right)\left\langle\mu_{s}^{N}, h_{s}\right\rangle d s+\widehat{\Lambda_{t}^{N}\left(F_{h}\right.}\right)+\left\langle\Lambda^{N}\left(F_{h}\right), \widetilde{\Lambda}^{N}\left(f_{G}\right)\right\rangle_{t},
$$

where $\left\{\widehat{\Lambda_{t}^{N}\left(F_{h}\right)}\right\}_{t \geq 0}$ is a local martingale under $\widehat{\mathbb{P}}_{\phi, G}^{N}$ with

$$
\left[\widehat{\Lambda^{N}\left(F_{h}\right)}, \widehat{\Lambda^{N}\left(F_{h}\right)}\right]=\left[\Lambda^{N}\left(F_{h}\right), \Lambda^{N}\left(F_{h}\right)\right] .
$$


Then, by Lemma 4.4 and Doob's inequality, $\left.\widehat{\Lambda_{t}^{N}\left(F_{h}\right.}\right)=o_{p}(1)$ under $\widehat{\mathbb{P}}_{\phi, G}^{N}$ and hence

$$
\left\langle\mu_{t}^{N}, h_{t}\right\rangle=\left\langle\mu_{0}^{N}, h_{0}\right\rangle+\int_{0}^{t}\left(\partial_{s}+\mathcal{L}_{N}\right)\left\langle\mu_{s}^{N}, h_{s}\right\rangle d s+o_{p}(1)+\left\langle\Lambda^{N}\left(F_{h}\right), \widetilde{\Lambda}^{N}\left(f_{G}\right)\right\rangle_{t}
$$

under $\widehat{\mathbb{P}}_{\phi, G}^{N}$.

Next we calculate $\left\langle\Lambda^{N}\left(F_{h}\right), \widetilde{\Lambda}^{N}\left(f_{G}\right)\right\rangle_{t}$. According to the definition of $\widetilde{\Lambda}_{t}^{N}\left(f_{G}\right)$ and (4.2),

$$
d\left\langle\Lambda^{N}\left(F_{h}\right), \widetilde{\Lambda}^{N}\left(f_{G}\right)\right\rangle_{t}=\frac{1}{f_{G}\left(t, \eta_{t}\right)} d\left\langle\Lambda^{N}\left(F_{h}\right), \Lambda^{N}\left(f_{G}\right)\right\rangle_{t},
$$

where

$$
d\left\langle\Lambda^{N}\left(F_{h}\right), \Lambda^{N}\left(f_{G}\right)\right\rangle_{t}=\left(\mathcal{L}_{N}\left(F_{h} f_{G}\right)-f_{G} \mathcal{L}_{N} F_{h}-F_{h} \mathcal{L}_{N} f_{G}\right) d t
$$

By direct calculations,

$$
\frac{1}{f_{G}}\left(\mathcal{L}_{N}\left(F_{h} f_{G}\right)-f_{G} \mathcal{L}_{N} F_{h}-F_{h} \mathcal{L}_{N} f_{G}\right)=\mathrm{I}_{N}+\mathrm{II}_{N}
$$

where

$$
\begin{aligned}
\mathrm{I}_{N}= & \frac{N^{2}}{a_{N}} \sum_{i \neq-1}\left(\eta_{t}(i+1)-\eta_{t}(i)\right)\left(h_{t}\left(\frac{i}{N}\right)-h_{t}\left(\frac{i+1}{N}\right)\right) \\
& \times\left(\exp \left\{\frac{a_{N}}{N}\left(\eta_{t}(i+1)-\eta_{t}(i)\right)\left(G_{t}\left(\frac{i}{N}\right)-G_{t}\left(\frac{i+1}{N}\right)\right)\right\}-1\right)
\end{aligned}
$$

and

$$
\begin{aligned}
\mathrm{II}_{N}= & \frac{N}{a_{N}}\left(\eta_{t}(0)-\eta_{t}(-1)\right)\left(h_{t}\left(\frac{-1}{N}\right)-h_{t}\left(\frac{0}{N}\right)\right) \\
& \times\left(\exp \left\{\frac{a_{N}}{N}\left(\eta_{t}(0)-\eta_{t}(-1)\right)\left(G_{t}\left(\frac{-1}{N}\right)-G_{t}\left(\frac{0}{N}\right)\right)\right\}-1\right) .
\end{aligned}
$$

By Taylor's expansion formula up to second order,

$$
\mathrm{I}_{N}=\frac{1}{N} \sum_{i \neq-1}\left(\eta_{t}(i)-\eta_{t}(i+1)\right)^{2} \partial_{u} h_{t}\left(\frac{i}{N}\right) \partial_{u} G_{t}\left(\frac{i}{N}\right)+o(1)
$$

and

$$
\mathrm{II}_{N}=\left(\eta_{t}(-1)-\eta_{t}(0)\right)^{2}\left(h_{t}(0)-h_{t}(1)\right)\left(G_{t}(0)-G_{t}(1)\right)+o(1)
$$

Since $\left(\eta_{t}(i)-\eta_{t}(i+1)\right)^{2}=\eta_{t}(i)\left(1-\eta_{t}(i+1)\right)+\eta_{t}(i+1)\left(1-\eta_{t}(i)\right)$, Lemmas 2.1 and 2.2 control the errors when we replace $\left(\eta_{t}(i)-\eta_{t}(i+1)\right)^{2}$ by $2 \rho(1-\rho)$ in $\mathrm{I}_{N}$ and $\mathrm{II}_{N}$. To be precise, under $\mathbb{P}_{\rho}^{N}$

$$
\int_{0}^{T} \mathrm{I}_{N} d t=2 \rho(1-\rho) \int_{0}^{T} \int_{0}^{1} \partial_{u} h_{t}(u) \partial_{u} G_{t}(u) d u d t+o(1)+o_{\exp }\left(a_{N}\right)
$$

and

$$
\int_{0}^{T} \mathrm{II}_{N} d t=2 \rho(1-\rho) \int_{0}^{T}\left(h_{t}(0)-h_{t}(1)\right)\left(G_{t}(0)-G_{t}(1)\right) d t+o(1)+o_{\exp }\left(a_{N}\right) .
$$


By Taylor's expansion formula, it is not difficult to show that there exists a finite constant $C$ independent of $N$ such that $\frac{d \nu_{N, \phi}}{d \nu_{\rho}} \leq e^{C a_{N}}$ for sufficiently large $N$. Therefore, $\frac{d \widehat{\mathbb{P}}_{\phi, G}^{N}}{d \mathbb{P}_{\rho}^{N}} \leq e^{C a_{N}}$ for large $N$. By Cauchy-Schwartz inequality, Equations (4.6) and (4.7) also hold under $\widehat{\mathbb{P}}_{\phi, G}^{N}$. As a result, under $\widehat{\mathbb{P}}_{\phi, G}^{N}$,

$$
\begin{aligned}
& \left\langle\mu_{t}^{N}, h_{t}\right\rangle=o_{p}(1)+\left\langle\mu_{0}^{N}, h_{0}\right\rangle+\int_{0}^{t}\left(\partial_{s}+\mathcal{L}_{N}\right)\left\langle\mu_{s}^{N}, h_{s}\right\rangle d s \\
& +2 \rho(1-\rho)\left(\int_{0}^{t}\left(h_{s}(0)-h_{s}(1)\right)\left(G_{s}(0)-G_{s}(1)\right) d s+\int_{0}^{t} \int_{0}^{1} \partial_{u} h_{s}(u) \partial_{u} G_{s}(u) d u d s\right) .
\end{aligned}
$$

Now we calculate $\left(\partial_{s}+\mathcal{L}_{N}\right)\left\langle\mu_{s}^{N}, h_{s}\right\rangle$. By direct calculations,

$$
\begin{aligned}
\mathcal{L}_{N}\left\langle\mu_{s}^{N}, h_{s}\right\rangle= & \frac{N^{2}}{a_{N}} \sum_{i=0}^{N-1}\left(\eta_{s}(i)-\rho\right)\left(h_{s}\left(\frac{i+1}{N}\right)+h_{s}\left(\frac{i-1}{N}\right)-2 h_{s}\left(\frac{i}{N}\right)\right) \\
& +\frac{N-N^{2}}{a_{N}}\left(\eta_{s}(0)-\eta_{s}(-1)\right)\left(h_{s}\left(\frac{-1}{N}\right)-h_{s}(0)\right)=\mathrm{III}_{N}+\mathrm{IV}_{N},
\end{aligned}
$$

where

$$
\mathrm{III}_{N}=\frac{N^{2}}{a_{N}} \sum_{i \neq 0,-1}\left(\eta_{s}(i)-\rho\right)\left(h_{s}\left(\frac{i+1}{N}\right)+h_{s}\left(\frac{i-1}{N}\right)-2 h_{s}\left(\frac{i}{N}\right)\right)
$$

and

$$
\begin{aligned}
\operatorname{IV}_{N}= & \frac{N}{a_{N}}\left(\eta_{s}(0)-\eta_{s}(-1)\right)\left(h_{s}\left(\frac{-1}{N}\right)-h_{s}(0)\right)+\frac{N^{2}}{a_{N}}\left(\eta_{s}(0)-\rho\right)\left(h_{s}\left(\frac{1}{N}\right)-h_{s}(0)\right) \\
& +\frac{N^{2}}{a_{N}}\left(\eta_{s}(-1)-\rho\right)\left(h_{s}\left(\frac{-2}{N}\right)-h_{s}(-1)\right) .
\end{aligned}
$$

By Taylor's expansion formula up to third order,

$$
\operatorname{III}_{N}=\left\langle\mu_{s}^{N}, \tilde{\Delta} h_{s}\right\rangle+o(1)
$$

Since $h \in \mathscr{G}$, it is not difficult to check that $\operatorname{IV}_{N}=o(1)$.

In conclusion, we have shown that under $\widehat{\mathbb{P}}_{\phi, G}^{N}$,

$$
\begin{aligned}
& \left\langle\mu_{t}^{N}, h_{t}\right\rangle=o_{p}(1)+\left\langle\mu_{0}^{N}, h_{0}\right\rangle+\int_{0}^{t}\left\langle\mu_{s}^{N},\left(\partial_{s}+\tilde{\Delta}\right) h_{s}\right\rangle d s \\
& +2 \rho(1-\rho)\left(\int_{0}^{t}\left(h_{s}(0)-h_{s}(1)\right)\left(G_{s}(0)-G_{s}(1)\right) d t+\int_{0}^{t} \int_{0}^{1} \partial_{u} h_{s}(u) \partial_{u} G_{s}(u) d u d s\right) \\
& =o_{p}(1)+\left\langle\mu_{0}^{N}, h_{0}\right\rangle+\int_{0}^{t}\left\langle\mu_{s}^{N},\left(\partial_{s}+\tilde{\Delta}\right) h_{s}\right\rangle d s+\int_{0}^{t}\left\langle h_{s} \mid G_{s}\right\rangle d s .
\end{aligned}
$$

Specially, when $h \in \mathscr{G}_{0}$,

$$
\left\langle\mu_{t}^{N}, h\right\rangle=o_{p}(1)+\left\langle\mu_{0}^{N}, h\right\rangle+\int_{0}^{t}\left\langle\mu_{s}^{N}, \tilde{\Delta} h\right\rangle d s+\int_{0}^{t}\left\langle h \mid G_{s}\right\rangle d s
$$


for all $0 \leq t \leq T$. Note that although the $o_{p}(1)$ term in the above equation is given for each $t$, it is easy to check that this $o_{p}(1)$ term can be chosen uniformly for $0 \leq t \leq T$. Since

$$
\mu_{t}^{G}(h)=\int_{0}^{1} \phi(u) h(u) d u+\int_{0}^{t} \mu_{s}^{G}(\tilde{\Delta} h) d s+\int_{0}^{t}\left\langle h \mid G_{s}\right\rangle d s
$$

by Grownwall's inequality,

$$
\left|\left\langle\mu_{t}^{N}, \theta_{m}\right\rangle-\mu^{G}\left(\theta_{m}\right)\right| \leq\left(o_{p}(1)+\left|\int_{0}^{1} \phi(x) \theta_{m}(x) d x-\left\langle\mu_{0}^{N}, \theta_{m}\right\rangle\right|\right) e^{\left|e_{m}\right| t}
$$

for all $0 \leq t \leq T$ and $m \geq 1$.

Therefore, to show that $\mu^{N}$ converges in $\widehat{\mathbb{P}}_{\phi, G}^{N}$-probability to $\mu^{G}$ in $D([0, T], \mathscr{M})$, we only need to show that

$$
\left\langle\mu_{0}^{N}, h\right\rangle=\int_{0}^{1} \phi(u) h(u) d u+o_{p}(1)
$$

under $\widehat{\mathbb{P}}_{\phi, G}^{N}$ for any $h \in \mathscr{G}_{0}$. According to the definition of $\nu_{N, \phi}$ and Chebyshev's inequality, it is easy to check that Equation (4.8) holds under $\mathbb{P}_{\phi}^{N}$. Since $M_{0}^{N}(G)=1, \mu_{0}^{N}$ has the same distribution under $\mathbb{P}_{\phi}^{N}$ and $\widehat{\mathbb{P}}_{\phi, G}^{N}$. This finishes the proof.

Proof of the lower bound. If $\inf _{\mu \in O} I(\mu)=+\infty$, then Equation (1.7) holds trivially. So we only need to deal with the case where $\inf _{\mu \in O} I(\mu)<+\infty$. For given $\epsilon>0$, there exists $\mu^{\epsilon} \in O$ such that

$$
I_{i n i}\left(\mu_{0}^{\epsilon}\right)+I_{d y n}\left(\mu^{\epsilon}\right) \leq \inf _{\mu \in O} I(\mu)+\epsilon .
$$

By Lemma 4.1, there exists $\phi^{\epsilon} \in L^{2}[0,1]$ and $\psi^{\epsilon} \in \mathscr{H}$ such that

$$
\mu_{0}^{\epsilon}(G)=\left\langle\phi^{\epsilon}, G\right\rangle, \forall G \in \mathscr{G}_{0}, \quad I_{\text {ini }}\left(\mu_{0}^{\epsilon}\right)=\frac{\int_{0}^{1}\left(\phi^{\epsilon}(u)\right)^{2} d u}{2 \rho(1-\rho)},
$$

and

$$
\ell_{T}\left(\mu^{\epsilon}, G\right)=\left\langle\left\langle G, \psi^{\epsilon}\right\rangle\right\rangle, \forall G \in \mathscr{G}, \quad I\left(\mu^{\epsilon}\right)=\frac{1}{2}\left\langle\left\langle\psi^{\epsilon}, \psi^{\epsilon}\right\rangle\right\rangle
$$

Let $G \in \mathscr{G}_{\text {such that }} G_{t}=b_{t} h$ for some $h \in \mathscr{G}_{0}$ and $b \in C^{1}[0, T]$. By the above formula and $(1.5)$,

$$
b_{T} \mu_{T}^{\epsilon}(h)-b_{0} \mu_{0}^{\epsilon}(h)-\int_{0}^{T} b^{\prime}(s) \mu_{s}^{\epsilon}(h) d s=\int_{0}^{T} b(s)\left(\mu_{s}^{\epsilon}(\tilde{\Delta} h)+\left\langle h \mid \psi_{s}^{\epsilon}\right\rangle\right) d s .
$$

Since $b$ is arbitrary, according to the formula of integration by parts, $\left\{\mu_{t}^{\epsilon}(h)\right\}_{0 \leq t \leq T}$ is absolutely continuous and

$$
\left\{\begin{array}{l}
\frac{d}{d t} \mu_{t}^{\epsilon}(h)=\mu_{t}^{\epsilon}(\tilde{\Delta} h)+\left\langle h \mid \psi_{t}^{\epsilon}\right\rangle \\
\mu_{0}^{\epsilon}(h)=\int_{0}^{1} \phi^{\epsilon}(u) h(u) d u
\end{array}\right.
$$

for any $h \in \mathscr{G}_{0}$.

Since $\mathscr{G}_{0}$ is dense in $L^{2}[0,1]$ by Lemma 1.1 and $\mathscr{G}$ is dense in $\mathscr{H}$, there exist $\phi_{n} \in \mathscr{G}_{0}$ and $\psi_{n} \in \mathscr{G}$ such that $\phi_{n}$ converges to $\phi^{\epsilon}$ in $L^{2}[0,1]$ and $\psi_{n}$ converges to $\psi^{\epsilon}$ in $\mathscr{H}$ as $n \rightarrow \infty$. Let $\mu_{n} \in D([0, T], \mathscr{M})$ such that $\mu_{n, 0}(G)=\left\langle\phi_{n}, G\right\rangle$ for any $G \in \mathscr{G}_{0}$, and $\ell_{T}\left(\mu_{n}, G\right)=\left\langle\left\langle G, \psi_{n}\right\rangle\right\rangle$ for 
any $G \in \mathscr{G}$. According to an analysis similar with that leading to Equation (4.9), $\mu_{n}$ is the solution to the Equation

$$
\left\{\begin{array}{l}
\frac{d}{d t} \mu_{n, t}(h)=\mu_{n, t}(\tilde{\Delta} h)+\left\langle h \mid \psi_{n, t}\right\rangle \\
\mu_{n, 0}(h)=\int_{0}^{1} \phi_{n}(u) h(u) d u
\end{array}\right.
$$

for any $h \in \mathscr{G}_{0}$. By Lemma 4.1,

$$
I_{i n i}\left(\mu_{n, 0}\right)=\frac{\int_{0}^{1}\left(\phi_{n}(u)\right)^{2} d u}{2 \rho(1-\rho)}
$$

and

$$
I_{d y n}\left(\mu_{n}\right)=\frac{1}{2}\left\langle\left\langle\psi_{n}, \psi_{n}\right\rangle\right\rangle=\ell_{T}\left(\mu_{n}, \psi_{n}\right)-\frac{1}{2}\left\langle\left\langle\psi_{n}, \psi_{n}\right\rangle\right\rangle .
$$

By (4.9), (4.10) and Grownwall's inequality, for any $0 \leq t \leq T$ and any integer $k$,

$$
\left|\mu_{n, t}\left(\theta_{k}\right)-\mu_{t}^{\epsilon}\left(\theta_{k}\right)\right| \leq\left|\int_{0}^{1}\left(\phi^{\epsilon}(u)-\phi_{n}(u)\right) \theta_{k}(u) d u+\left\langle\left\langle\theta_{k}, \psi^{\epsilon}-\psi^{n}\right\rangle\right\rangle\right| e^{\left|e_{k}\right| t} .
$$

Consequently, $\mu_{n}$ converges to $\mu^{\epsilon}$ in $D([0, T], \mathscr{M})$ and

$$
\lim _{n \rightarrow+\infty}\left(I_{d y n}\left(\mu_{n}\right)+I_{i n i}\left(\mu_{n, 0}\right)\right)=I_{d y n}\left(\mu^{\epsilon}\right)+I_{i n i}\left(\mu_{0}^{\epsilon}\right) .
$$

Hence, there exists $m \geq 1$ such that $\mu_{m} \in O$ and

$$
I_{d y n}\left(\mu_{m}\right)+I_{i n i}\left(\mu_{m, 0}\right) \leq I_{d y n}\left(\mu^{\epsilon}\right)+I_{i n i}\left(\mu_{0}^{\epsilon}\right)+\epsilon .
$$

Let $D_{\epsilon}=\left\{\mu:\left|\ell_{T}\left(\mu, \psi_{m}\right)-\ell_{T}\left(\mu_{m}, \psi_{m}\right)\right|<\epsilon\right\} \bigcap O$, then by Lemma 4.2 and Equation (4.10), $\mu^{N}$ converges in $\widehat{\mathbb{P}}_{\phi_{m}, \psi_{m}}^{N}$-probability to $\mu_{m}$ as $N \rightarrow+\infty$ and hence

$$
\lim _{N \rightarrow+\infty} \widehat{\mathbb{P}}_{\phi_{m}, \psi_{m}}^{N}\left(\mu^{N} \in D_{\epsilon}\right)=1
$$

According to the expression of $M_{T}^{N}(G)$ given in Equation (3.7) and Lemmas 2.1 and 2.2,

$$
M_{T}^{N}\left(\psi_{m}\right)=\exp \left\{\frac{a_{N}^{2}}{N}\left(\ell_{T}\left(\mu^{N}, \psi_{m}\right)-\frac{1}{2}\left\langle\left\langle\psi_{m}, \psi_{m}\right\rangle\right\rangle+o(1)+\widehat{\varepsilon}_{N}\right)\right\},
$$

where $\widehat{\varepsilon}_{N}=o_{\exp }\left(a_{N}\right)$ under $\mathbb{P}_{\rho}^{N}$. As we have shown above, $\frac{d \widehat{\mathbb{P}}_{\phi_{m}, \psi_{m}}^{N}}{d \mathbb{P}_{\rho}^{N}} \leq e^{C a_{N}}$ for sufficiently large $N$, hence $\widehat{\varepsilon}_{N}=o_{\text {exp }}\left(a_{N}\right)$ under $\widehat{\mathbb{P}}_{\phi_{m}, \psi_{m}}^{N}$.

According to the definition of $\nu_{N, \phi_{m}}$, Chebyshev's inequality and Taylor's expansion formula up to second order, it is not difficult to show that

$$
\frac{d \mathbb{P}_{\rho}^{N}}{d \mathbb{P}_{\phi_{m}}^{N}}=\exp \left\{-\frac{a_{N}^{2}}{N}\left(\frac{\int_{0}^{1} \phi_{m}^{2}(u) d u}{2 \rho(1-\rho)}+\widetilde{\varepsilon}_{N}\right)\right\}=\exp \left\{-\frac{a_{N}^{2}}{N}\left(I_{i n i}\left(\mu_{m, 0}\right)+\widetilde{\varepsilon}_{N}\right)\right\}
$$

where $\widetilde{\varepsilon}_{N}=o_{p}(1)$ under $\widehat{\mathbb{P}}_{\phi_{m}, \psi_{m}}^{N}$. Consequently, let

$$
\widehat{D}_{N, \epsilon}=\left\{\mu^{N} \in D_{\epsilon}\right\} \bigcap\left\{\left|\widehat{\varepsilon}_{N}\right|<\epsilon,\left|\widetilde{\varepsilon}_{N}\right|<\epsilon\right\},
$$


then

$$
\lim _{N \rightarrow+\infty} \widehat{\mathbb{P}}_{\phi_{m}, \psi_{m}}^{N}\left(\widehat{D}_{N, \epsilon}\right)=1
$$

For sufficiently large $N$, on $\widehat{D}_{N, \epsilon}$,

$$
\begin{aligned}
& \quad \frac{d \mathbb{P}_{\rho}^{N}}{d \widehat{\mathbb{P}}_{\phi_{m}, \psi_{m}}^{N}}=\frac{d \mathbb{P}_{\rho}^{N}}{d \mathbb{P}_{\phi_{m}}^{N}} \frac{d \mathbb{P}_{\phi_{m}}^{N}}{d \widehat{\mathbb{P}}_{\phi_{m}, \psi_{m}}^{N}}=\frac{d \mathbb{P}_{\rho}^{N}}{d \mathbb{P}_{\phi_{m}}^{N}} \frac{1}{M_{T}^{N}\left(\psi_{m}\right)} \\
& \geq \exp \left\{-\frac{a_{N}^{2}}{N}\left(I_{i n i}\left(\mu_{m, 0}\right)+\ell_{T}\left(\mu_{m}, \psi_{m}\right)-\frac{1}{2}\left\langle\left\langle\psi_{m}, \psi_{m}\right\rangle\right\rangle+3 \epsilon\right)\right\} \\
& =\exp \left\{-\frac{a_{N}^{2}}{N}\left(I_{i n i}\left(\mu_{m, 0}\right)+I_{d y n}\left(\mu_{m}\right)+3 \epsilon\right)\right\} \geq \exp \left\{-\frac{a_{N}^{2}}{N}\left(I_{i n i}\left(\mu_{0}^{\epsilon}\right)+I_{d y n}\left(\mu^{\epsilon}\right)+4 \epsilon\right)\right\} \\
& \geq \exp \left\{-\frac{a_{N}^{2}}{N}\left(\inf _{\mu \in O}\left(I_{i n i}\left(\mu_{0}\right)+I_{d y n}(\mu)\right)+5 \epsilon\right)\right\} .
\end{aligned}
$$

Therefore, by Equation (4.11),

$$
\begin{aligned}
& \liminf _{N \rightarrow \infty} \frac{N}{a_{N}^{2}} \log Q_{\rho}^{N}[O]=\liminf _{n \rightarrow+\infty} \frac{N}{a_{N}^{2}} \log \mathbb{P}_{\rho}^{N}\left(\mu_{N} \in O\right) \geq \liminf _{n \rightarrow+\infty} \frac{N}{a_{N}^{2}} \log \mathbb{P}_{\rho}^{N}\left(\widehat{D}_{N, \epsilon}\right) \\
& =\liminf _{n \rightarrow+\infty} \frac{N}{a_{N}^{2}} \log \widehat{\mathbb{E}}_{\phi_{m}, \psi_{m}}^{N}\left[\frac{d \mathbb{P}_{\rho}^{N}}{d \widehat{\mathbb{P}}_{\phi_{m}, \psi_{m}}^{N}} \mathbf{1}_{\widehat{D}_{N, \epsilon}}\right] \geq-\inf _{\mu \in O}\left(I_{i n i}\left(\mu_{0}\right)+I_{d y n}(\mu)\right)-5 \epsilon .
\end{aligned}
$$

Since $\epsilon$ is arbitrary, the proof is complete.

\section{A Appendix}

\section{A.1 Lemma 1.1}

Proof of Lemma 1.1. We use $e_{-n}$ to denote $-(2 \pi n)^{2}$ for $n \geq 0$ and use $e_{n}$ to denote $-k_{n}^{2}$ for $n \geq 1$. Let

$$
\widehat{\mathscr{G}_{0}}=\left\{G \in C^{2}[0,1]: G^{\prime}(0)=G^{\prime}(1)=G(0)-G(1)\right\},
$$

then, by direct calculations, $\left\{e_{n}\right\}_{-\infty<n<+\infty}$ are all the eigenvalues of $\tilde{\Delta}$ limited on $\widehat{\mathscr{G}_{0}}$. Moreover, $\theta_{-n}(x)=\cos (2 n \pi x)$ is the eigenvector with respect to $e_{-n}$ and $\theta_{n}(x)=\sin \left(k_{n}\left(x-\frac{1}{2}\right)\right)$ is the eigenvector with respect to $e_{n}$.

According to the definition of the operator $\frac{d}{d x} \frac{d}{d W}$ introduced in [9], $\left.\tilde{\Delta}\right|_{\hat{\mathscr{G}}_{0}}=\frac{d}{d x} \frac{d}{d W}$ for $W$ equal to Lebesgue measure plus the Dirac measure at 0. Consequently, by [9, Theorem 1], $\left\{\theta_{n}\right\}_{-\infty<n<+\infty}$ is an orthogonal basis of $L^{2}[0,1]$.

\section{A.2 Equation (2.6)}

Proof of Equation (2.6). By Fubini's theorem,

$$
\mathrm{E}_{\nu_{\rho}}\left[\left(\eta^{M, \mathrm{R}}(0)-\rho\right)^{2 k}\right]=\int_{0}^{+\infty} 2 k t^{2 k-1} \mathrm{P}_{\nu_{\rho}}\left(\left|\eta^{M, \mathrm{R}}(0)-\rho\right| \geq t\right) d t
$$


For $0 \leq t<1-\rho$ and $\theta \geq 0$, by Chebyshev's inequality,

$$
\begin{aligned}
\mathrm{P}_{\nu_{\rho}}\left(\eta^{M, \mathrm{R}}(0)-\rho \geq t\right) & \leq e^{-t M \theta} \mathrm{E}_{\nu_{\rho}}\left[e^{\theta M\left(\eta^{M, \mathrm{R}}(0)-\rho\right)}\right]=\left(e^{-\theta t} \mathrm{E}_{\nu_{\rho}}\left[e^{\theta(\eta(0)-\rho)}\right]\right)^{M} \\
& =\left(e^{-\theta(t+\rho)}\left[e^{\theta} \rho+1-\rho\right]\right)^{M} .
\end{aligned}
$$

Let $\theta=\log \frac{(t+\rho)(1-\rho)}{\rho(1-t-\rho)}$, then

$$
\mathrm{P}_{\nu_{\rho}}\left(\eta^{M, \mathrm{R}}(0)-\rho \geq t\right) \leq e^{-M \mathscr{I}(t)}
$$

for $0 \leq t<1-\rho$, where

$$
\mathscr{I}(t)=-(1-t-\rho) \log (1-\rho)+(t+\rho) \log (t+\rho)+(1-t-\rho) \log (1-\rho-t)-(t+\rho) \log \rho .
$$

We define $\mathscr{I}(1-\rho)=-\log \rho$. Note that $\lim _{t \uparrow 1-\rho} \mathscr{I}(t)=-\log \rho$ and

$$
\mathrm{P}_{\nu_{\rho}}\left(\eta^{\mathrm{M}, \mathrm{R}}(0)-\rho \geq 1-\rho\right)=\left(\mathrm{P}_{\nu_{\rho}}(\eta(0)=1)\right)^{\mathrm{M}}=\rho^{\mathrm{M}},
$$

hence Equation (A.1) holds for $0 \leq t \leq 1-\rho$ and $\mathscr{I}$ is continuous in $[0,1-\rho]$. It is easy to check that $\mathscr{I}(0)=0$ and $\mathscr{I}(t)>0$ for $t \in(0,1-\rho]$. By L'Hospital's rule,

$$
\lim _{t \downarrow 0} \frac{\mathscr{I}(t)}{t^{2}}=\frac{1}{2 \rho(1-\rho)} .
$$

Hence $\frac{\mathscr{I}(t)}{t^{2}}$ is continuous and strictly positive on $[0,1-\rho]$. Let $J_{1}(\rho)=\inf _{0 \leq t \leq 1-\rho} \frac{\mathscr{I}(t)}{t^{2}}$, which is strictly positive, then

$$
\mathrm{P}_{\nu_{\rho}}\left(\eta^{M, \mathrm{R}}(0)-\rho \geq t\right) \leq e^{-M J_{1}(\rho) t^{2}}
$$

for all $M \geq 1$ and any $t \in[0,1-\rho]$ by Equation (A.1). Note that $\mathrm{P}_{\nu_{\rho}}\left(\eta^{M, \mathrm{R}}(0)-\rho \geq t\right)=0$ for $t>1-\rho$, hence Equation (A.2) holds for all $M \geq 1$ and $t \geq 0$. A similar argument proves that there exists $J_{2}(\rho)>0$ such that

$$
\mathrm{P}_{\nu_{\rho}}\left(\eta^{M, \mathrm{R}}(0)-\rho \leq-t\right) \leq e^{-M J_{2}(\rho) t^{2}}
$$

for all $M \geq 1$ and $t \geq 0$. Let $J(\rho)=\inf \left\{J_{1}(\rho), J_{2}(\rho)\right\}$, then

$$
\begin{aligned}
\mathrm{E}_{\nu_{\rho}}\left[\left(\eta^{M, \mathrm{R}}(0)-\rho\right)^{2 k}\right] & =\int_{0}^{+\infty} 2 k t^{2 k-1} P_{\nu_{\rho}}\left(\left|\eta^{M, \mathrm{R}}(0)-\rho\right| \geq t\right) d t \\
& \leq \int_{0}^{+\infty} 4 k t^{2 k-1} e^{-M J(\rho) t^{2}} d t=\frac{2 k !}{M^{k}(J(\rho))^{k}} .
\end{aligned}
$$

Equation (2.6) follows by taking $C(\rho)=\frac{2}{J(\rho)}$.

\section{A.3 Existence and Uniqueness of solution to Equation (4.3)}

Proof of the existence. We directly construct a solution to Equation (4.3). For $-\infty<n<+\infty$, let $\left\{x_{t}^{n}\right\}_{0 \leq t \leq T}$ be the unique solution to the ODE

$$
\left\{\begin{array}{l}
\frac{d}{d t} x_{t}^{n}=e_{n} x_{t}^{n}+\left\langle\theta_{n} \mid G_{t}\right\rangle \\
x_{0}^{n}=\int_{0}^{1} \phi(x) \theta_{n}(x) d x
\end{array}\right.
$$


where $e_{n}$ is defined as in the proof of Lemma 1.1. That is to say,

$$
x_{t}^{n}=e^{e_{n} t} \int_{0}^{1} \phi(x) \theta_{n}(x) d x+\int_{0}^{t} e^{e_{n}(t-s)}\left\langle\theta_{n} \mid G_{s}\right\rangle d s .
$$

For any $f=\sum_{-\infty<n<+\infty} C_{n}(f) \theta_{n} \in \mathscr{G}_{0}$ and $t \geq 0$, we define

$$
\mu_{t}^{G}(f)=\sum_{-\infty<n<+\infty} C_{n}(f) x_{t}^{n} .
$$

Note that the coefficients $\left\{C_{n}(f)\right\}_{-\infty<n<+\infty}$ are unique according to Lemma 1.1 and hence the definition of $\mu^{G}$ is reasonable. Since

$$
\mu_{t}^{G}\left(\theta_{n}\right)=x_{t}^{n} \quad \text { and } \quad \mu_{t}^{G}\left(\tilde{\Delta} \theta_{n}\right)=\mu_{t}^{G}\left(e_{n} \theta_{n}\right)=e_{n} x_{t}^{n},
$$

it is easy to check that $\mu^{G}$ is the solution to Equation (4.3).

Proof of the uniqueness. Assuming that $\mu$ and $\nu$ are both solutions to Equation (4.3), then

$$
\left|\mu_{t}\left(\theta_{n}\right)-\nu_{t}\left(\theta_{n}\right)\right| \leq\left|e_{n}\right| \int_{0}^{t}\left|\mu_{s}\left(\theta_{n}\right)-\nu_{s}\left(\theta_{n}\right)\right| d s
$$

By Grownwall's inequality,

$$
\left|\mu_{t}\left(\theta_{n}\right)-\nu_{t}\left(\theta_{n}\right)\right| \leq 0 e^{\left|e_{n}\right| t}=0
$$

for any $0 \leq t \leq T$ and $n \geq 1$. Hence, $\mu=\nu$ and the proof is complete.

Acknowledgments. The authors are grateful to the financial support from the National

Natural Science Foundation of China with grant numbers 11501542 and 11971038.

\section{References}

[1] R. Baldasso, O. Menezes, A. Neumann, and R. Souza. Exclusion process with slow boundary. Journal of Statistical Physics, 167(5):1112-1142, 2017.

[2] A. A. Borovkov. Probabilities of large deviations in topological spaces, I. Siberian Mathematical Journal, 19:697-709, 1978.

[3] A. de Acosta and X. Chen. Moderate deviations for empirical measures of markov chains. Journal of Theoretical Probability, 11:1075-1110, 1998.

[4] A. Dembo and O. Zeitouni. Large Deviations: Techniques and Applications. Springer, 1997.

[5] T. Franco, P. Gonçalves, and A. Neumann. Hydrodynamical behavior of symmetric exclusion with slow bonds. In Annales de l'IHP Probabilités et statistiques, volume 49, pages 402-427, 2013.

[6] T. Franco, P. Gonçalves, and A. Neumann. Phase transition in equilibrium fluctuations of symmetric slowed exclusion. Stochastic Processes and their Applications, 123(12):41564185, 2013. 
[7] T. Franco, P. Gonçalves, and A. Neumann. Phase transition of a heat equation with Robins boundary conditions and exclusion process. Transactions of the American Mathematical Society, 367(9):6131-6158, 2015.

[8] T. Franco, P. Gonçalves, and G. M. Schütz. Scaling limits for the exclusion process with a slow site. Stochastic Processes and their applications, 126(3):800-831, 2016.

[9] T. Franco and C. Landim. Hydrodynamic limit of gradient exclusion processes with conductances. Archive for Rational Mechanics and Analysis, 195:409-439, 2009.

[10] T. Franco and A. Neumann. Large deviations for the exclusion process with a slow bond. The Annals of Applied Probability, 27(6):3547-3587, 2017.

[11] T. Franco and M. Tavares. Hydrodynamic limit for the SSEP with a slow membrane. Journal of Statistical Physics, 175(2):233-268, 2019.

[12] F. Q. Gao. Moderate deviations for martingales and mixing random processes. Stochastic Processes and their Applications, 61:263-275, 1996.

[13] F. Q. Gao and J. Quastel. Moderate deviations from the hydrodynamic limit of the symmetric exclusion process. Science in China Series A: Mathematics, 46(5):577-592, 2003.

[14] C. Kipnis and C. Landim. Scaling limits of interacting particle systems, volume 320. Springer Science \& Business Media, 2013.

[15] H. Nikaidô. On a minimax theorem and its applications to functional analysis. Journal of the Mathematical Society of Japan, 5(1):86-94, 1953.

[16] V. J. Schuppen and E. Wong. Transformation of local martingales under a change of law. The Annals of Probability, 2:879-888, 1974.

[17] F. Y. Wang, J. Xiong, and L. H. Xu. Asymptotics of sample entropy production rate for stochastic differential equations. Journal of Statistical Physics, 163:1211-1234, 2016.

[18] R. Wang and T. S. Zhang. Moderate deviations for stochastic reaction-diffusion equations with multiplicative noise. Potential Analysis, 42:99-113, 2015.

[19] L. Wu. Moderate deviations of dependent random variables related to CLT. The Annals of Probability, 23:420-445, 1995. 